\title{
Individual Premotor Drive Pulses, Not Time-Varying Synergies, Are the Units of Adjustment for Limb Trajectories Constructed in Spinal Cord
}

\author{
William J. Kargo ${ }^{1,2}$ and Simon F. Giszter ${ }^{1}$ \\ ${ }^{1}$ Department of Neurobiology and Anatomy, Drexel University College of Medicine, Philadelphia, Pennsylvania 19129, and ${ }^{2}$ Neurocrine Biosciences Inc., \\ San Diego, California 92130
}

\begin{abstract}
Complex actions may arise by combining simple motor primitives. Our studies support individual premotor drive pulses or bursts as execution primitives in spinal cord. Alternatively, the fundamental execution primitives at the segmental level could be time-varying synergies. To distinguish these hypotheses, we examined sensory feedback effects during targeted wiping organized in spinal cord. This behavior comprises three bursts. We tested (1) whether feedback altered the structure of individual premotor drive bursts or primitives, and (2) whether feedback differentially modulated different drive bursts or pulses in the three burst sequence. At least two of the three bursts would need to always be comodulated to support a time-varying synergy. We used selective muscle vibration to control spindle feedback from a single muscle (biceps/iliofibularis). The structures of premotor drive bursts were conserved. However, biceps vibration (1) scaled the amplitudes of two bursts coactivated during the initial phase of wiping independently of one another without altering their phase, and (2) independently phase regulated the third burst but preserved its amplitude. Thus, all three bursts were regulated separately. Durations were unaffected. The independent effects depended on (1) time of vibration during wiping, (2) frequency of vibration, and (3) limb configuration. Because each of the three bursts was independently modulated, these data strongly support execution using individual premotor bursts rather than time-varying synergies at the spinal level of motor organization. Our data show that both sensory feedback and central systems of the spinal cord act in concert to adjust the individual premotor bursts in support of the straight and unimodal wiping trajectory.
\end{abstract}

Key words: primitive; force-field; spinal cord; time-varying synergy; reflex; proprioceptive feedback; premotor

\section{Introduction}

The vertebrate nervous system may generate limb movement by combining simple building blocks, synergies, or primitives (Robertson et al., 1985; Berkinblit et al., 1986; Bizzi et al., 1991, 1995; Giszter et al., 1993; Smith and Stein, 1997; Stein et al., 1998; Raasch and Zajac, 1999; Tresch and Bizzi, 1999; Tresch et al., 1999; Kargo and Giszter, 2000a; Hart and Giszter, 2004; Lemay and Grill, 2004; Sosnik et al., 2004; Flash and Hochner, 2005; Cappellini et al., 2006; d'Avella et al., 2006; Torres-Oviedo et al., 2006). Several kinds of primitives have been suggested (Poppele and Bosco, 2003; Flash and Hochner, 2005; Bizzi et al., 2007; Giszter et al., 2007). We have proposed that fixed-duration bursts or pulses of premotor drive (and associated viscoelastic force fields) are the fundamental execution elements or primitives for spinal movement construction (Giszter et al., 1993, 2007; Kargo and Giszter, 2000a; Hart and Giszter, 2004). In such bursts, mus-

Received April 10, 2007; revised Dec. 21, 2007; accepted Dec. 23, 2007.

This work was supported by National Institutes of Health Grant NS40412 (S.F.G.) and Training Grant HD07467 (to Dr. Marion Murray) and by an Allegheny Singer Research Institute graduate student award (W.J.K.). We are very grateful to Drs. Michel Lemay, Corey Hart, and Arun Ramakrishnan and anonymous reviewers for their comments.

Correspondence should be addressed to Simon F. Giszter, Neurobiology and Anatomy, Drexel University College of Medicine, 2900 Queen Lane, Philadelphia, PA 19129. E-mail: simon.giszter@drexel.edu.

DOI:10.1523/JNEUROSCI.3229-07.2008

Copyright $\odot 2008$ Society for Neuroscience $\quad$ 0270-6474/08/282409-17\$15.00/0 cles must covary proportionally and synchronously. The premotor drive bursts may relate to the pattern shaping layers in spinal pattern generation suggested by McCrea and colleagues (Lafreniere-Roula and McCrea, 2005; Quevedo et al., 2005; McCrea and Rybak, 2007). An alternative perspective is the idea of time-varying synergies. These comprise sequential muscle activation patterns, in which activity of muscles need not be synchronous. Such sequences forming time-varying synergies are also proposed to be modulated and used as compositional units (d'Avella and Bizzi, 2005; d'Avella et al., 2006; Bizzi et al., 2007). Examining adjustments of motor patterns in spinal cord may distinguish between these schemes.

The frog's hindlimb wipe uses a directed trajectory with many of the characteristics of voluntary limb trajectories seen in man (Giszter et al., 1989; Schotland and Rymer, 1993; Sergio and Ostry, 1993). The kinematic path is nearly straight, and the tangential velocity is bell shaped. The motor pattern driving this trajectory comprises three bursts in the absence of corrections (Kargo and Giszter, 2000a,b). This provides an interesting framework to test these alternative ideas.

For spinal primitives to simplify organization of movement for descending controls, the interactions of descending and segmental reflex controls should preserve the fundamental modular structures. In mammals and other tetrapods, many descending 
controls act on the same interneuronal systems engaged by proprioception (e.g., corticospinal projections to group Ia and Ib interneurons). In a spinal frog, the descending controls have been lesioned. However, proprioceptive and cutaneous controls can be manipulated in various ways (Kargo and Giszter, 2000a; Giszter and Kargo, 2002). The proprioceptive adjustments that are possible for the motor pattern of the main wiping sequence have not been thoroughly examined.

Proprioceptive feedback could have several kinds of effects on the wiping sequence. For control purposes, the three bursts could be organized into five possible groupings: (1) a single timevarying synergy of three bursts, (2) three patterns composed of time-varying-synergy burst pairs and a complementary single burst, or finally (3) as three single bursts. If basic burst structure was preserved but feedback independently modulated the amplitudes and the timing of the bursts in the pattern, this would thus argue strongly against time-varying synergies as representing the fundamental building blocks in spinal cord. Similarly, if feedback altered the balance or synchrony of muscle activity in individual bursts acting as units, the resulting force-field structure would differ (Fig. 2 summarizes these tests) (Giszter et al., 2007). Such a result would seriously challenge the notion of unit premotor drive bursts as a set of simple, invariant building blocks or primitives for constructing reflex trajectories and behaviors. Experiments here thus provide strong tests of both schemes.

Our results show that feedback preserved the pulsed premotor drive structures and associated force-field structures. However, the feedback independently modified the phasing and amplitude of the three different bursts in the motor sequence. Our data support the idea that independent premotor bursts and not timevarying synergies are the units of composition and adjustment at the spinal level.

\section{Materials and Methods}

\section{Surgery}

Adult bullfrogs ( $200-350 \mathrm{~g})$ were anesthetized with $0.5 \mathrm{cc}$ of $5 \%$ Tricaine (dissolved in frog Ringer's solution) applied subcutaneously. The spinal cord was transected at the medullary-spinal cord junction (for details, see Kargo and Giszter, 2000a). Vertebral laminas 6-10 were removed, and the dura was opened to expose the lumbar spinal cord and provide access to dorsal roots 7-9 ipsilateral to the wiping limb. These dorsal roots carry sensory information from receptors of the ipsilateral hindlimb to the spinal cord. Gelfoam was placed over the exposed cord and roots, and Ringer's solution-soaked cotton was placed over the exposed back muscles. Skin incisions were closed with wound clips.

We used seven-strand Teflon-coated stainless steel wires with $0.7 \mathrm{~mm}$ exposure tipped by a small wax or plastic ball, which was used to retain the intramuscular electrode in position in vivo. Pairs of electrodes were implanted in the following muscles: biceps (BI), vastus externus (VE), rectus anterior (RA), semimembranosus (SM), gluteus (GL), ventral head of semitendinosus (ST), adductor (AD), rectus internus (RI) and iliopsoas (IL). "Reverse patch" electrodes were used with the sheet-like sartorius muscle (SA) (for a description, see Kargo and Giszter, 2000a). Sites of implantation were the same between frogs. Lombard and Abbott (1907), Loeb et al. (1999), and Kargo and Rome (2002) described hindlimb muscle actions in the frog. BI and SA are multiarticular muscles that flex the knee and hip. VE is multiarticular muscle with a large extensor moment at the knee and a small hip flexor moment. RA is a multiarticular muscle that extends the knee and flexes the hip. SM and RI are multiarticular muscles with large extensor moments at the hip and small flexor moments at the knee. GL is a uniarticular muscle that primarily abducts and externally rotates the hip. The ventral ST head is a biarticular muscle that flexes the knee and extends the hip. AD is uniarticular hip extensor (HE). IL is a uniarticular hip flexor (HF). After implanting muscles, the EMG wires were braided together and led out of the incision. Skin inci- sions were closed with wound clips and cyanoacrylate tissue adhesive (Vetbond; 3M, St. Paul, MN).

On the ventral surface of the ankle (of the wiping limb), a small incision was made to expose the lower tibiofibula, including the malleolus. After removing fascia, bone screws were placed in the malleolus and 3-4 $\mathrm{mm}$ superior to the malleolus. Bone screws were coated with carboxylate cement (Durelon, Norristown, PA), and a steel standoff $(0.62 \mathrm{~cm}$ outer diameter, $2-3 \mathrm{~cm}$ in length) was placed in the cement. A metal attachment was screwed into the standoff. The attachment was secured into an ankle restraint that was connected to a six-axis force/torque transducer (gamma 3/10, 12 bit, range, $\pm 2.27 \mathrm{~kg}$; ATI Instruments, Garner, NC). During isometric wiping trials, hip and knee torques were transmitted through the bones of the limb to the force sensor via the standoff/metal attachment on the ankle. There was thus no intervening transmission of force through the skin and soft tissue. Skin impacts have been shown to evoke correction responses during wiping (Kargo and Giszter, 2000a). Care was taken to place the bone screws and standoff at similar positions in all frogs. Frogs were placed in a refrigerator at $10^{\circ} \mathrm{C}$ overnight after the surgery.

\section{Wiping preparation}

On the following day, the frog was placed on a molded stand (gama 3/10, 12 bit, range, $\pm 2.27 \mathrm{~kg}$; that supported the body and contralateral (target) hindlimb in the horizontal plane. The pelvis and vertebral column were immobilized using custom-made clamps. Hindlimb-to-hindlimb wiping was evoked by electrically stimulating the dorsolateral surface of the heel of the target (or contralateral) limb. The stimulation was delivered via bipolar leads (2-3 mm separation) and consisted of a $600 \mathrm{~ms}$ train of $3-8 \mathrm{~V}, 2 \mathrm{~ms}$ biphasic pulses applied at $30-40 \mathrm{~Hz}$. The stimulus evoked a pattern of movement in the wiping limb in which the ankle moved to the midline, contacted the stimulus on the target limb, and attempted to remove it with a whisking motion. As in previous studies (Giszter et al., 1993; Giszter and Kargo, 2000; Kargo and Giszter, 2000a), the wiping limb was then restrained in the force sensor arrangement described above to record isometric forces generated at the ankle. The wiping limb was positioned and restrained in the same horizontal plane as the target limb.

The tendon of the BI in the wiping limb was detached at its distal insertion on the tibiofibula below the knee joint. A small hook was placed through the tendon. Suture was used to further secure the tendon around the hook. Fascia between BI and adjacent muscles was removed, and exposed muscles were covered with a Vaseline-mineral oil mix to prevent them from drying. The hook in the distal tendon of BI was tightly secured into a 3.5 inch, $8 \Omega$ speaker mounted apart from the air table on which the preparation was positioned. This arrangement was used to vibrate BI and to precisely recruit muscle spindles (Giszter and Kargo, 2002).

In all experiments, BI was paralyzed by injecting $0.05 \mathrm{cc}$ of $10 \mu \mathrm{M}$ D-tubocurarine chloride near the two motor endpoints of the muscle nerve. Curare paralysis thus served to avoid uncontrolled effects of spindle drive causing intrafusal contractions and prevented extrafusal contractions in the vibrated muscle. Intrafusal contractions would alter spindle sensitivity to vibration, and extrafusal contractions would transiently alter the mechanics of the vibrated muscle, making precise vibration control difficult (Giszter and Kargo, 2002). BI was enveloped by Vaseline to prevent muscle drying and possible spread of curare to adjacent muscles. The curare dosage eliminated BI EMG (monitored on oscilloscope) and muscle contraction. Forces recorded at the ankle and EMGs were monitored on a trial-by-trial basis to determine whether other muscles were affected by the curare. We also determined that $\beta$-skeletofusimotor innervation was blocked in BI by our curare dosage. Spindles were identified by standard means as described by Giszter and Kargo (2002), using ventral root stimulation to generate a brief pause in firing. Single-unit recordings in some frogs showed that muscle spindles from the uncurarized $\mathrm{BI}$ fired at an average frequency of $75 \mathrm{~Hz}$ during isometric wiping responses. Curarization reduced this to a static level of $0-10 \mathrm{~Hz}$, depending on the holding length of BI. The curare-paralyzed spindle afferents still generate time-locked action potentials in response to vibration of the muscle. These have been shown to precisely track vibration up to $\sim 120$ $\mathrm{Hz}$ (Giszter and Kargo, 2002). The magnetic mount of the speakers was 
separate from and mechanically isolated from the air table on which the frog was mounted to prevent resonant transmission of vibration through that table to the vertebral or other clamps. The speaker was positioned so that the BI muscle belly was $\sim 30^{\circ}$ above the horizontal plane. BI was held at the length the muscle would rest in vivo, at the configuration the limb was positioned (hip angle, $\sim 0^{\circ}$ relative to mediolateral axis of frog; internal knee angle, $\sim 110^{\circ}$ ). In vivo length was measured with calipers before tendon detachment. Two infrared emitting diodes (IREDs) were placed on the proximal and distal ends of BI to monitor its length during vibration with an optical position sensor (Optotrak; Northern Digital, Waterloo, Ontario, Canada) (see below, Data collection and basic data analysis). A function generator was used to sinusoidally drive the speaker at different frequencies $(20-110 \mathrm{~Hz})$. Measured length changes in the muscle were sinusoidal, along the long axis of the muscle and $\sim 150-200$ $\mu \mathrm{m}$ (as reported by Giszter and Kargo, 2002). We used $1 \mathrm{kHz}$ collection and moving averages filtering of OPTOTRAK data from our air-tableisolated muscles to make these measurements.

\section{Data collection and basic data analysis}

Isometric forces at the ankle, EMGs from 10 wiping limb muscles, single afferent units from exposed dorsal roots, and BI length were recorded in all wiping trials. Forces were recorded using a six-axis force/torque transducer. EMG signals were led to a differential amplifier (A-M Systems, Everett, WA) for bandpass filtering $(100 \mathrm{~Hz}$ and $10 \mathrm{kHz})$ and amplified 1000 times. Single afferent units were recorded from the exposed dorsal roots using tungsten electrodes (5-8 $\mathrm{M} \Omega$ impedance at $1 \mathrm{kHz}$ ) and suction electrodes from A-M Systems. The suction or microelectrode recording and ground leads were led to a differential amplifier where the signals were bandpass filtered (cutoffs, $100 \mathrm{~Hz}$ and $10 \mathrm{kHz}$ ) and amplified 10,000 times. Analog recordings were analog-to-digital converted using a Das16 converter and Mega FIFO memory board. EMGs were sampled at $10 \mathrm{kHz}$ per channel. However, subsequently, the EMG was rectified and low-pass filtered. Forces were sampled at $250 \mathrm{~Hz}$, and each was stored on the same computer using custom-written software. Three-dimensional position data of the IREDs were sampled at $1 \mathrm{kHz}$ and stored on computer using Northern Digital software. The distance between the diodes (i.e., BI muscle length) was calculated during speaker vibration. Length recordings were synchronized to EMG, force, and single-unit recordings via a computer-generated trigger.

For each wiping trial, the direction and magnitude of the forces that were produced at the ankle (referred to here as endpoint forces), in the horizontal plane of the limb (mediolateral axis, right $0^{\circ}$ and left $180^{\circ}$; longitudinal axis, nose $90^{\circ}$ and cloaca $270^{\circ}$ ), were determined by the following:

$$
\begin{aligned}
\text { direction } & =\arctan _{2} \frac{F_{y}(t)}{F_{x}(t)} \\
\text { magnitude } & =\sqrt{F_{y}^{2}(t)+F_{x}^{2}(t)},
\end{aligned}
$$

where $F_{y}$ and $F_{x}$ are the force components in the horizontal plane over time $(t)$. Force direction and magnitude were determined for the active forces, i.e., when the resting, baseline forces were subtracted from total forces generated during a wiping trial.

Force fields were constructed as described in detail by Giszter et al. (1993), and force-field comparisons were made as described by MussaIvaldi et al. (1994) and Kargo and Giszter (2000a).

For each trial, the static joint torques that were produced at the hip and knee were determined from the endpoint forces, limb position, and link lengths by the following:

$$
\begin{array}{r}
{\left[\begin{array}{c}
T_{H} \\
T_{K}
\end{array}\right]=\left[\begin{array}{cc}
-l_{t} \sin \Theta_{h}-l_{c} \sin \left(\Theta_{h}+\Theta_{k}\right) & l_{t} \cos \Theta_{h}+l_{c} \cos \left(\Theta_{h}+\Theta_{k}\right) \\
-l_{c} \sin \left(\Theta_{h}+\Theta_{k}\right) & l_{c} \cos \left(\Theta_{h}+\Theta_{k}\right)
\end{array}\right]} \\
\cdot\left[\begin{array}{c}
F_{x} \\
F_{y}
\end{array}\right],(3
\end{array}
$$

where $l_{t}$ and $l_{c}$ are lengths of the thigh and calf, $\Theta_{h}$ and $\Theta_{k}$ are the hip and knee angles using the measurement convention by Asada and Slotine (1986), and $T_{H}$ and $T_{K}$ are the hip and knee torques acting in the hori- zontal plane of the limb, i.e., omitting elevator and rotator hip torques, which act out of this plane.

For each trial, EMGs were rectified and filtered using a 30-point moving average (Kargo and Giszter, 2000b). The area under the EMG envelope of each muscle, the peak EMG amplitude, time of peak amplitude, times of EMG onset and offset, and the latency between the timing features of each muscle were determined for each trial.

\section{Experimental series and controls}

For most experiments, vibration began at wipe onset and continued for either 200 or $600 \mathrm{~ms}$. We examined spindle frequency effects by applying vibration continuously for $600 \mathrm{~ms}$ starting at onset, through the placing phase (the trajectory portion) of the wipe, thereby avoiding timing variation. This strategy highlighted changes that could be induced throughout the placing phase of the wipe. We examined effects of spindle bursts and their timing in more detail by applying vibration (1) only during the initial burst of wiping ( $200 \mathrm{~ms}$, beginning at wipe onset) or (2) by applying brief fixed-frequency $(100 \mathrm{~Hz})$ and fixed-duration $(100 \mathrm{~ms})$ bursts of vibration at various predetermined times. These $100 \mathrm{~ms}$ vibration bursts thus occurred briefly during different motor bursts in the placing phase. We examined effects of configuration of the limb on spindle input gain or gating by altering limb configuration while again applying vibration at a fixed frequency continuously for $600 \mathrm{~ms}$ through the placing phase of the wipe. This avoided effects of timing variations, and we once more examined alterations that could be induced throughout the placing phase of the wipe.

As baseline data and controls, we first tested whether our vibration of the biceps muscles selectively activated only BI afferents and not other muscle afferents. Spread elsewhere could be attributable to either (1) spread of vibration from the biceps muscle through its intact origin (at the hip) to other muscles or (2) imperfect air-table isolation between the speaker mount and the preparation. We tested vibration effects with, and then without, neural feedback from the target muscle in one frog. First, we recorded wiping forces (when BI was detached) under two conditions: with and without vibration applied for the duration of the wiping response. The effects attributable to vibration are described in Results. At the end of the experiment, the motor nerve to BI was cut. The frog was then allowed to rest for several hours to allow any injury discharge to subside (Kargo and Giszter, 2000b), and wiping was then evoked under the two conditions. If the effects attributable to vibration persisted, this would be evidence that vibration affected afferents in addition to or other than BI afferents. If the effects were absent, this would be evidence that vibration activated only (functional) feedback pathways from BI afferents. The vibration effects reported here disappeared after the BI nerve cut, which supports the selective activation of only BI afferents by our vibration method.

Three basic experiments comprised the body of this study. These experiments tested whether and how different regimens of BI vibration affected the wiping motor pattern (both the isometric forces and EMGs). The series are described below and shown in Figure 1.

Throughout the subsequent experiments, BI was held curarized at the in vivo length in our standard test configuration. The absence of any contraction also eliminated the phasic firing rates of tension receptors. This low level of tonic activity provided a baseline on which various patterns of activity were imposed by vibration.

Frequency-dependent effects ( 13 frogs). BI was vibrated at a range of frequencies at a single-limb configuration. Ten to 15 wiping trials were elicited at one of four or five different frequencies (no vibration, 10, 30, 70 , and $110 \mathrm{~Hz}$ ). Vibration was triggered at the onset of the wiping motor pattern (i.e., at onset of the ST EMG, which was always the first detected). In eight frogs, vibration was applied only for the duration of the first motor phase (for $\sim 200 \mathrm{~ms}$ ), before the onset of hip extensor activation. In a second group of five frogs, vibration was applied for the duration of the first two motor phases (for $\sim 600 \mathrm{~ms}$ ) and therefore throughout the entire placing phase of the wipe. In both groups, frequency-dependent effects of vibration were examined. The ranges of frequencies used were in the biologically observed range (Kargo, 2000).

Phase-dependent effects (six frogs). BI was vibrated in brief bursts at specific times during the wiping response in six frogs. This patterning was 
A

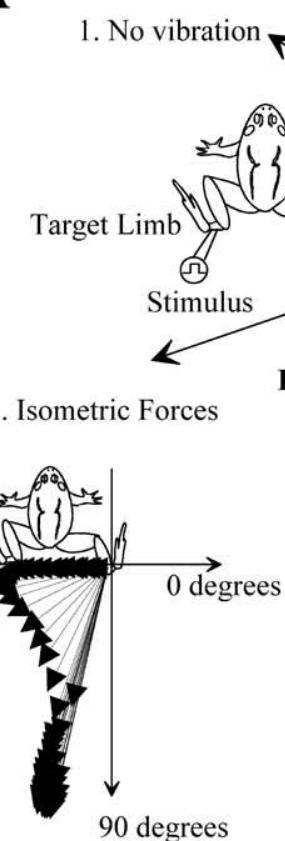

CONDITIONS

2. Vibration of

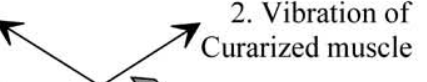

B

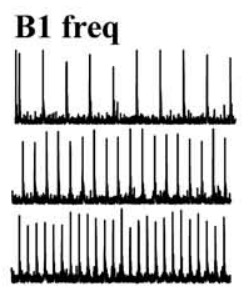

B3 limb configuration

A2. Isometric EMG records

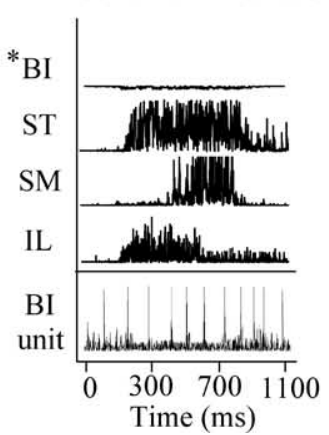

B2 burst timing

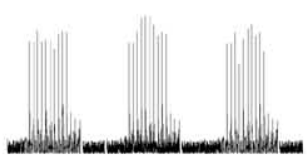

B4 holding BI length
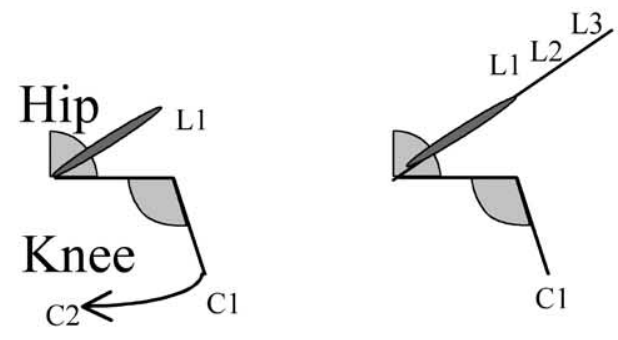

Figure 1. In this study, we examined the organization of heteronymous feedback effects from the BI during hindlimb-to-hindlimb wiping. $A$, Wiping was evoked by stimulating the foot of the target limb. Isometric forces were recorded at the ankle of the wiping limb. EMGs were recorded from 10 muscles in the wiping limb. BI was detached at its distal insertion and secured to a vibrator. The length of the BI muscle was recorded by an optical position sensor to monitor the frequency and amplitude of the applied vibration. The effect of biceps vibration on wiping forces at the ankle and electromyogram activity in the motor pattern were examined. An example of the two data types are shown below the frog: $\boldsymbol{A 1}$, force vectors generated at the ankle and their magnitude and directions referenced to the mediolateral axis; $\boldsymbol{A}$, rectified EMGs from three muscles. $\boldsymbol{B}$, In this study, four different vibration paradigms were used during wiping trials (B1-B4). We tested how varying vibration frequency (B1) and varying onset and duration of vibration bursts (B2) affected the wiping force and motor patterns. We tested how varying the time of onset of brief (100 ms) periods of vibration affected the wiping force and motor patterns (B2). We tested whether varying limb configuration altered the effects of a constant frequency of vibration at a constant biceps length (B3). We tested how varying the holding length of $\mathrm{BI}$, when a constant vibration frequency was applied, affected the wiping force and motor patterns (B4).

closer to the physiologically observed behavior of frog spindles in wiping than continuous activity. The bursts were $100 \mathrm{~ms}$ in duration, and the frequency was $100 \mathrm{~Hz}$. The bursts were applied at different times spanning the interval from $200 \mathrm{~ms}$ before ST EMG onset up to motor pattern offset. Thirty to 50 wiping trials were evoked in individual frogs. Previously, we showed with spindle recordings (Giszter and Kargo, 2002) that, during the bursts of vibration, spindles reliably fired in a 1:1 manner relative to the vibration frequency (i.e., $10 \pm 1$ spikes for the $100 \mathrm{~ms}$ duration of vibration). Ex vivo studies of ST muscle spindles showed that some spindles may fire prolonged, plateau-like discharges to brief mechanical stimuli (Sokabe et al., 1993). Such plateau-like firing patterns were not observed in any of the BI muscle spindles recorded here, under in vivo conditions (Kargo, 2000).

Configuration-dependent effects (seven frogs). BI was vibrated at a range of limb configurations in seven frogs. The lengths of other muscles and presumably the tonic and phasic discharge rates of their muscle afferents will vary across the configuration space of the limb. However, BI was kept at a fixed length at each of the tested limb positions. At each position, wiping was evoked without and with $100 \mathrm{~Hz}$ BI vibration. Vibration was triggered at ST onset and lasted for the duration of the first two motor phases ( $\sim 600 \mathrm{~ms}$ ). Trials were evoked with the wiping limb held at 11-12 different positions that spanned the reachable workspace of the limb.

Control experiment 1: damage to other sensory afferents. Before curarizing and vibrating BI, we examined whether tendon detachment might have altered sensory feedback from other afferents (e.g., by nerve damage). In each frog tested here, wiping trials were evoked when BI was still attached to the tibia and when the distal tendon was detached, secured to the vibrator, and held at its in vivo length. Feedback from BI and other afferents should be similar in both instances, i.e., similar isometric contractions.

Control experiment 2: damage to motor nerves or effectors. The detachment procedure may have damaged motor nerves or altered the aponeuroses and mechanics of muscles adjacent to BI. To test this, we performed the following experiment. First, wiping forces were recorded in the intact limb. Second, BI was stimulated through its indwelling EMG electrodes $(0.5 \mathrm{~V}, 40 \mathrm{~Hz}, 1 \mathrm{~ms}$ biphasic pulses). The endpoint forces generated by BI contraction were determined (total forces - passive forces). Third, the distal tendon of $\mathrm{BI}$ was detached and secured to the vibrator, and wiping forces were recorded in the absence of the BI contribution. Because the endpoint forces generated by individual muscles sum at the ankle (Mussa-Ivaldi et al., 1994), the only observed difference between conditions (if EMGs are the same; see above) should be the absence of forces contributed by BI. Vector subtraction of the wiping forces was performed and is represented by the following:

$$
F_{\text {res }}(r, t)=F_{I}(r, t)-F_{R}(r, t)
$$

where $F_{I}(r, t)$ represents the forces generated with BI intact, and $F_{R}(r, t)$ represents the forces generated after removal of $\mathrm{BI}$ contributions. We examined whether the direction of $F_{r e s}(r, t)$ was the same direction as $F_{B I}(r, t)$, which represents the forces generated by (evoked) BI contraction in the intact limb.

Data analysis framework: defining a primitive and possible feedback effects

Because we will present muscle pattern and isometric force pattern changes and because several kinds of primitives can be envisioned, we developed an analysis framework able to distinguish different kinds of primitives and the conditions for rejecting them based on muscle pattern and/or force pattern changes. These are detailed below and in the supplemental Appendix (available at www.jneurosci.org as supplemental material) for the reader concerned with the technical issues.

To organize our analysis of heteronymous feedback effects within the primitive framework, we first formalize our definitions of a primitive, of the muscle contributions to a primitive, of the wiping motor pattern, and of feedback effects. These definitions are presented fully in the supple- 


\section{A Force-field primitive / premotor drive tests}

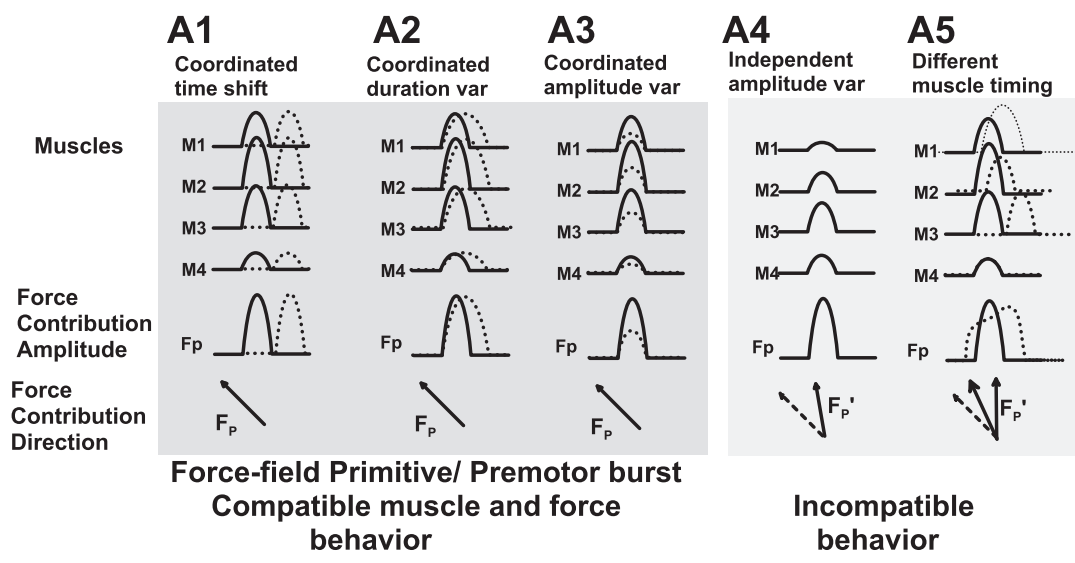

\section{B Time-varying synergy tests in wiping}

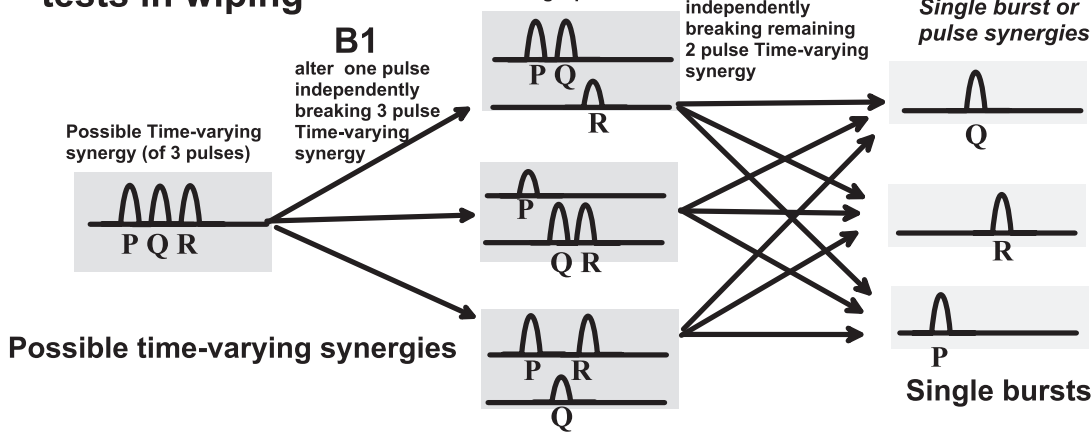

Figure 2. Hypothesis testing of premotor burst/force-field primitives and time-varying synergies. $A$, Force-field primitive premotor drive tests. Dark gray, Hypothesis preserving; light gray, hypothesis rejecting. A1, Coordinated time shift of a synchronous burst of a group of muscles, preserving muscle balance, will preserve the force direction at each configuration (solid to dotted). $\mathbf{A 2}$, Coordinated duration changes preserving muscle balance will preserve force direction at each configuration (solid to dotted). $\mathbf{A}$, Coordinated amplitude changes preserving ratios of muscles activity will preserve force direction at each configuration (solid to dotted). Mechanisms $\mathbf{A} \mathbf{1 - A 3}$ in combination also preserve force orientation at a configuration. $\boldsymbol{A 4}$, Independent amplitude modulation or variation will not preserve force direction, would not be extracted by independent components analyses as a unitary drive, and does not match the force-field primitive/premotor drive burst hypothesis. (A4 amplitude ratios differ from dotted and solid in $\mathbf{A 3}$.) $\mathbf{A 5}$, Independent time shifts or dilations of bursts will not preserve force direction, would not be extracted by independent components analyses as a unitary drive, and does not match the force-field primitive/premotor drive burst hypothesis. B, Time-varying synergy tests (3-burst example). Dark gray, Hypothesis preserving; light gray, hypothesis rejecting. A three-burst system like the aiming trajectory of wiping is the simplest framework to test time-varying synergies directly. The bursts $P Q R$ could be a unit of three-burst time-varying synergy (left; $\{P, Q, R\}$ ) or three different groups of two-burst time-varying synergy $(\{P, Q\},\{Q, R\},\{P, R\} ;$ middle panels) and the remaining single-burst patterns. Finally (right), the bursts could be independent of one another. Two control tests $\boldsymbol{B} \mathbf{1}$ and $\mathbf{B} \mathbf{2}$ differentiate the three-burst, two-burst, and single-burst possibilities. Each test shows that one burst in a three- or two-burst group can be regulated independently by some manipulation of proprioceptive or descending feedback.

mental Appendix (available at www.jneurosci.org as supplemental material) and were also previously elaborated by Giszter et al. (2007). They are shown diagrammatically in Figure 2 .

We define a force-field primitive by the following:

$$
F(r, \dot{r}, t)=A \times a(t) \times \phi(r, \dot{r}),
$$

where $F(r, \dot{r}, t)$ represents the position $(r)$, and velocity $(\dot{r})$ represents dependent forces that are produced by a (conservative) primitive $\phi$ across the configuration and velocity space of the hindlimb and that are measured at the ankle in a scaled pulse defined by $a(t)$. This definition can support composition schemes as proposed by Mussa-Ivaldi (1992) and Mussa-Ivaldi and Giszter (1992). Under isometric conditions, $\dot{r}=0$, and Equation 6 is reduced to

$$
F(r, t)=A \times a(t) \times \phi(r),
$$

where $\phi(r)=\phi(r, 0), a(t)$ is the normalized time course (from 0 to 1 ), and $A$ is the peak amplitude, which is specific to position but also context.

Previous studies (Giszter et al., 1993; Kargo and Giszter, 2000a; Giszter and Kargo, 2001) showed that the isometric forces produced during hindlimb-to-hindlimb wiping could then be minimally described as follows:

$$
F(r, t)=\sum_{i}^{N} A_{i} \times a\left(t+\tau_{i}\right) \times \phi_{i}(r)
$$

as a sum of $i$ primitives of amplitude $A_{i}$ and onset $\tau_{i}$. We envision feedback from BI afferents could have several possible effects within this framework (see Fig. 2) (supplemental Appendix, available at www.jneurosci.org as supplemental material). First, the parameters $A_{i}, \tau_{i}$ or the time function $a$ could be altered (Fig. 2A1-A3), or, second, individual muscles within primitives could be regulated differentially (Fig. $2 A 4, A 5)$. These two types of effects divide cleanly into two groups of predictions (expressed as Equations A11-A15, A16-A19 in supplemental Appendix, available at www. jneurosci.org as supplemental material). The first set of effects (Fig. 2A1-A3) acts to scale the primitive or its time course but preserves the ankle force orientation. These effects are characterized by conserved force direction, proportional covariation of the activity of muscles in the primitive, and covariation of their onset times and durations. The second set of effects (Fig. 2A4,A5) alter the force directions of the primitive by providing unbalanced or nonuniform excitation-inhibition to the muscles forming the primitive. These latter differential effects would present a substantial challenge to the hypothesis of primitives even at the level of the isolated spinal cord. Thus, the experiments present a critical test of the hypothesis of forcefield primitives.

To distinguish these two possibilities above in conditions in which multiple primitives might overlap in time, we used a vector subtraction procedure initially introduced by Kargo and Giszter (2000a) and described above by Equation 4 . Here, the forces generated without vibration were subtracted from time-matched forces generated with different regimens of vibration. If the resultant forces produced by the subtraction have a fixed direction over time, this result would support the hypothesis that the net effect of biceps feedback (heteronymous) could be captured by one of the Equations A11-A15 of the supplemental Appendix (available at www. jneurosci.org as supplemental material) and were consistent with the hypothesis of primitives. We also examined EMGs (of muscles contributing to the primitive) to further confirm this. The supplemental Appendix (available at www.jneurosci.org as supplemental material) (Giszter et al., 2007) shows that the simplest way to preserve force directions in the force field is that EMGs should covary in a similar manner (along a single slope) between conditions. If the resultant forces have a rotating force direction over time, it would support the hypothesis that the biceps feedback caused differing muscle specific increases-decreases. In this case, EMGs (of muscles forming the 
primitive) would not covary in a similar manner between conditions. We tested this by plotting EMG waveforms against one another and finding the average slope of this relationship over time.

To test for timing differences among muscles forming a primitive (supplemental Appendix, Equations A12-A14, preserving force direction, or Equations A17-A19, changing force direction; available at www.jneurosci.org as supplemental material) after application of vibration, we compared the EMG onset times of muscles that contributed to the component primitives between conditions (to test for changes in $\tau_{i}$ ). We also compared the peak EMG magnitudes and times [to test for changes in $a(t)$ ]. Mean values were determined for multiple trials, and statistical differences between conditions were assessed using standard, matched $t$ tests implemented in S-Plus (a statistical software package from Mathsoft, Seattle, WA).

Finally, it is important to stress that the experimental series, which is detailed above (see Experimental series and controls), also goes beyond simple hypothesis testing and attempts to identify the frequency, time, configuration, and muscle-length dependency of the possible effects attributable to biceps feedback (e.g., amplitude scaling, phase regulation, time course variation, and unbalanced feedback regulation).

\section{Defining a time-varying synergy}

We here define a time-varying-synergy simply as "two or more pulses of muscle activity sequenced in time, which are regulated as a unit." Regulation could occur either by amplitude scaling, or phase shifting the unit, or by the temporal dilation of the units. We specify that there must be at least two pulses or bursts of muscle activity. We do this because a single pulse of synchronous muscle activity is identical to the idea of a premotor burst or a force-field primitive, and then the hypotheses are not different. Furthermore, time-varying synergies have been used as multi-burst units in this way (d'Avella and Bizzi, 2005; d'Avella et al., 2006). Note that force direction is not necessarily constrained in a time-varying synergy as it is in the premotor drive bursts. The force direction that is generated can potentially vary significantly as a single time-varying synergy executes, because there are no defined constraints on the covariation of amplitude or phase of muscles in individual bursts.

To test the idea of time-varying synergies, we use the observation that there are three bursts in the main sequence of wiping and call them $P, Q$, and $R$ (Fig. $2 B$ ).

Control could act on the following: (1) $\{P, Q, R\}$ as a unit; (2) $\{P, Q\}$ as a unit and $\{R\} ;(3)\{Q, R\}$ as a unit and $\{P\}$; or $(4)\{P, R\}$ as a unit and $\{Q\}$. If control acts on $\{P\},\{Q\}$, and $\{R\}$ separately and independently, then the pulses act as force-field primitives, not time-varying synergies. If any one pulse can be controlled separately, we can eliminate possibility 1 (Fig. 2 B, step B1). Suppose this were $\{R\}$. Option 2 remains viable. However, if a second pulse other can this first $\{R\}$ can be separately controlled (say, $\{P\}$ ) we split the former pairing $\{P, Q\}$ (as in Fig. $2 B$, step B2) and are thus forced to use a description using three independent pulses or premotor drive bursts and not a multi-pulse time-varying synergy.

Using these sets of tests, the vibration-induced changes in motor pattern can be used to tests both hypotheses of force-field primitives/premotor drive pulses and time-varying synergies in the spinal cord.

\section{Results}

We recorded the pattern of isometric forces that were generated at the ankle of the wiping limb and the underlying hindlimb EMGs in spinalized frogs (for setup, see Fig. 1A). These data formed the basis for subsequent analysis and experiments. Force and EMG patterns, when all muscles were intact, have been published previously (Giszter et al., 1993; Kargo and Giszter, 2000a; Giszter and Kargo, 2001). Here, the distal tendon of BI was detached from the wiping limb and secured to a vibrator. For the majority of the experiments, the BI muscle was paralyzed with curare (see Materials and Methods) to avoid uncontrolled effects of spindle drive and extrafusal contraction in the vibrated muscle. Before paralyzing BI and applying different regimens of vibration, we first performed experiments to determine BI contribu- tions to wiping force patterns and to ensure that our procedure of detaching BI did not significantly disrupt motor responses.

\section{$\mathrm{BI}$ detachment and $\mathrm{BI}$ contributions to the wiping response}

Disruption of motor behavior after detaching BI might occur as result of loss of sensory afference, damage to the motor nerves to adjacent muscles, or as a result of physical interactions among the adjacent muscles themselves. In each frog tested in this study, isometric wiping responses were evoked (1) when the distal tendon of BI was still attached to the tibiofibula and (2) when the distal tendon was detached and secured to a vibrator. After it was detached, the BI was held at its in vivo length. Thus, in the first set of wiping trials, BI contracted isometrically and directly contributed to forces recorded at the ankle. In the second set of trials, BI contracted isometrically (against the vibrator attachment) but did not contribute directly to forces recorded at the ankle. Feedback from BI afferents should be similar in both instances and, therefore, the behavior should not differ, i.e., similar isometric contractions in all muscles. The bottom of Figure $3 A$ shows averaged EMGs from five muscles in a single frog during isometric wiping trials with BI attached (bold EMGs) and with BI detached (thin, shaded EMGs). The averaged data were constructed from eight trials for each condition. Detachment of BI did not alter the amplitude or timing of EMGs during the initial motor phase (ST, $\mathrm{BI}$, and IL activation) or during the second motor phase, in which the hip extensors SM and RI were activated. Onset and termination of bursts were determined by persistent rise above or fall below 2 SDs from the mean quiescent EMG/noise level. Similarly, none of the frogs presented in the following sections showed significant changes in the magnitude or time of onset/offset of EMGs during wiping trials after detaching BI. Therefore, the detachment procedure did not cause any major changes in sensory feedback, which might arise from injury to adjacent sensory receptors in muscles or other tissues or to BI muscle receptors.

In addition to the control above, we considered the possibility that the detachment procedure may have altered the aponeuroses and mechanics of other muscles at the knee adjacent to the BI tendon or the behavior of unrecorded muscles. If this happened, the ankle force contribution would be altered from that predicted by force loss because of BI detachment alone. To examine this, we examined the pattern of wiping forces before and after detachment of BI. At the top of Figure $3 A$, individual force vectors for wiping trials with $\mathrm{BI}$ intact and with $\mathrm{BI}$ detached are plotted every $40 \mathrm{~ms}$. In the two-link (thigh and calf) system examined here, the forces generated by individual muscles that cross the hip and knee sum linearly at the ankle (Giszter et al., 1993; Mussa-Ivaldi et al., 1994; Loeb et al., 2000). Thus, if the force pattern was altered solely by the removal of BI forces, subtraction of the force vectors generated in BI-detached trials (B.D.) from time-matched forces generated in BI-intact (B.I.) trials should yield those forces contributed by BI alone. The forces produced by the vector subtraction are shown in the bottom row of the top panel of Figure $3 \mathrm{~A}$ (marked Res for resultant vectors). The averaged direction and magnitude of forces with BI intact (bold lines), BI detached (thin, solid lines), and for the resultant (dotted lines) are shown over time in the middle panel of Figure $3 A$. The resultant forces had a uniform direction $\left(165 \pm 5^{\circ}\right)$ over time. This can be seen in the distribution of the resultant force directions over the duration of wiping, which is shown as a polar histogram in Figure $3 B$ (top). In the bottom polar histogram, the distribution of the force directions produced by stimulation of BI for $600 \mathrm{~ms}$ duration, when intact in the hindlimb before its detachment, is shown. The two distributions have the same mean angle $\left(165^{\circ}\right)$, and both have a 

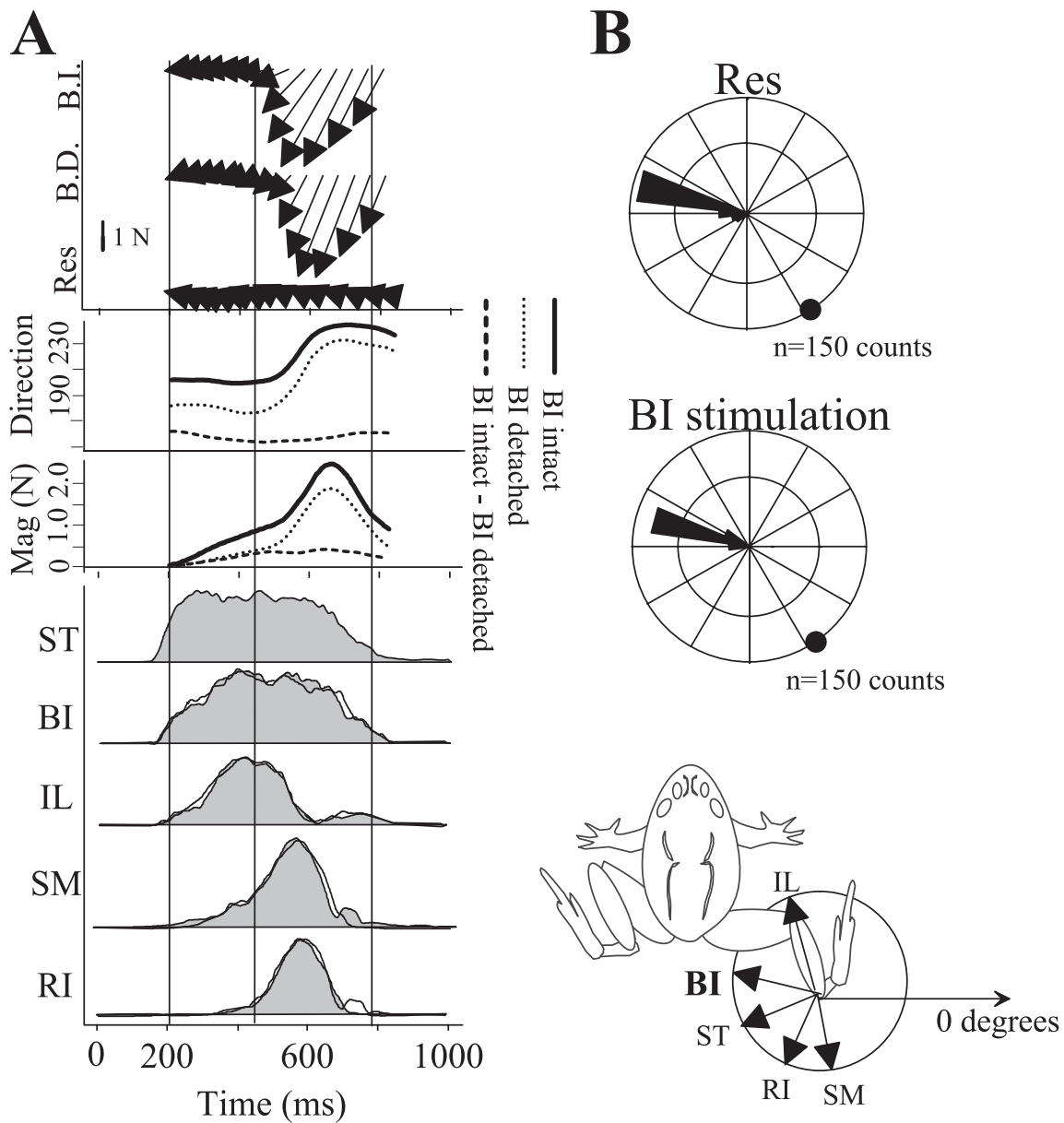

Figure 3. In each frog, wiping trials were evoked with the distal tendon of Bl still intact $(\boldsymbol{A})$ and with the tendon detached from the limb and secured to a vibrator $(\boldsymbol{B})$. In the first set of trials, BI contracted isometrically and contributed to forces at the ankle, and, in the second set, BI contracted isometrically against the vibrator. In the second set of trials, BI did not contribute to forces recorded at the ankle. Typical data are shown. $A$, The averaged force vectors produced during wiping trials in a single frog with $B$ muscle intact and attached (B.I.) and then with BI detached (B.D.) are shown at $40 \mathrm{~ms}$ intervals in the top two rows of the top panel. In the bottom row of this panel, force vectors produced by vector subtraction of B.D. forces from B.I. forces are shown (Res for resultant). In the middle two panels, the averaged magnitude and direction of B.D. (thin solid lines), B.I. (thick solid lines), and Res (dotted lines) forces are shown. Direction is referenced to the axis shown as $0^{\circ}$ in the bottom of Figure $2 B$. In the bottom panel, the rectified, filtered, and averaged EMGs for five muscles (ST, BI, IL, SM, and RI) are shown for B.D. (thin-lined shaded envelopes) and B.I. (thick-lined envelopes) trials. Averages were constructed from eight trials for each condition. BI detachment did not alter the motor pattern. The magnitude of wiping forces was reduced and the direction of forces altered after BI detachment. The resultant force vectors (i.e., the forces removed by BI detachment) were uniformly directed over time $\left(160^{\circ}\right)$. $\boldsymbol{B}$, We tested whether the direction of the resultant force vectors over time was similar to the direction of forces produced by direct stimulation of $\mathrm{BI}$ (i.e., when intact, before detaching its tendon). The polar histograms show the distribution of force directions resulting from subtraction of BD from BI forces over a wiping trial (a 600 ms duration; top histogram) and the distribution of force directions obtained over a $600 \mathrm{~ms}$ duration of Bl stimulation. Each bin represents $14.4^{\circ}$. The distributions were not significantly different (see Results). In the bottom panel, the mean direction produced by direct stimulation of the five muscles in $\boldsymbol{A}$ are shown. Force directions from stimulation of the BI, ST, RI, SM, and IL muscles in the limb configuration used here are diagrammed. Thus, detaching BI simply removes the $\mathrm{BI}$ force contribution at the ankle rather than compromising adjacent muscles or nerves.

low variance $\left(<12^{\circ}\right)$. These data indicate that the only force alteration after BI detachment was likely to be the removal of its contribution. Thus, this test provides strong evidence that the primary effect of detaching BI on the wiping force pattern was simply the subtraction of BI forces from forces expressed at the ankle and no other perturbation.

The BI detachment allowed us to asses the importance of BI contribution to ankle force directly. In the frogs used here, BI contributed on average to $33 \%$ of the total force during the first phase $(0.34 \mathrm{~N}$ of $1.03 \mathrm{~N}$ of force) and to $12 \%$ of the total force during the second phase $(0.30 \mathrm{~N}$ of $2.5 \mathrm{~N}$ of force). The force vector contributed by BI results from the hip and knee torques it produces transmitted by the mechanical linkage of the limb. For BI, the relative proportion of hip flexion to knee flexion torque is $0.5: 1.0 \mathrm{~N} / \mathrm{cm}$ at the reference limb configuration of $0^{\circ}$ hip flexion and $110^{\circ}$ knee flexion (Kargo and Rome, 2002).

\section{Amplitude scaling of a hip flexor primitive}

The preceding experiment established that the normal wiping motor pattern could be elicited in our paradigm. We then examined the effects of feedback from BI muscle spindles as they were entrained by muscle vibration applied during wiping. BI was curarized to eliminate its extrafusal muscle contraction and $\beta$-skeletofusimotor activation of muscle spindles. These two contraction effects would have made precise control of the activity of the BI spindles with vibration difficult. The extrafusal contractions would alter the mechanics of the vibrated muscle and vibration source. The intrafusal contractions would alter spindle response properties. In this experimental series, we evoked wiping in 10 frogs. As wiping was evoked, the BI was held fixed or vibrated at 30,70 , or $110 \mathrm{~Hz}$. Vibration was applied at the onset of wiping (ST EMG onset) and continued for a $200 \mathrm{~ms}$ duration. This represented approximately four-fifths of the duration of the first EMG burst composing the wiping motor pattern. The second EMG burst in hip extensor muscles began at 200-250 ms relative to ST onset. Thus, vibration was restricted to occur only during the first muscle burst.

Forces were generated in several overlapping pulses or waves in wiping with the leg held isometrically. Wave here refers to the wave-like time course of forces that is associated with each EMG burst composing the motor pattern. We first tested whether BI vibration affected the amplitude or direction of forces produced during the initial wave of wiping forces. We examined this at a single-limb configuration $\left(0^{\circ}\right.$ hip angle and $110^{\circ}$ internal knee angle). The top panel of Figure $4 A$ shows the averaged (12 trials) force vectors produced during wiping trials with no vibration, 30,70 , and $110 \mathrm{~Hz}$ vibration. The vectors were collected at $250 \mathrm{~Hz}$, but they are plotted every $40 \mathrm{~ms}$ for clarity. The magnitude and direction of these forces are shown in the middle panels (thin solid line, no vibration; thick solid line, 30 $\mathrm{Hz}$; dashed line, $70 \mathrm{~Hz}$; dotted line, $110 \mathrm{~Hz}$ ). Increased vibration frequencies led to increases in the amplitude of the initial wave of forces. Increasing vibration frequency also caused force direction changes, i.e., increased rotation toward the hip. The peak amplitude of this initial wave was $1.11 \mathrm{~N}$ at $110 \mathrm{~Hz}, 0.92 \mathrm{~N}$ at $70 \mathrm{~Hz}, 0.61$ $\mathrm{N}$ at $30 \mathrm{~Hz}$, and $0.51 \mathrm{~N}$ with no vibration. The direction at the 


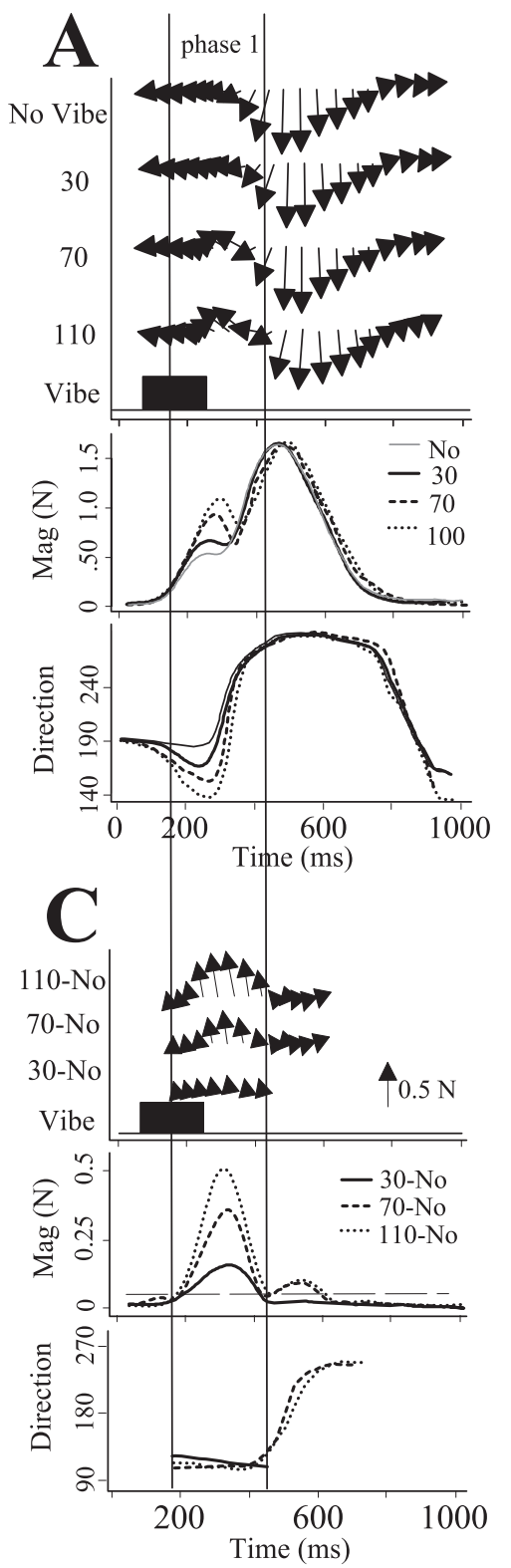

B
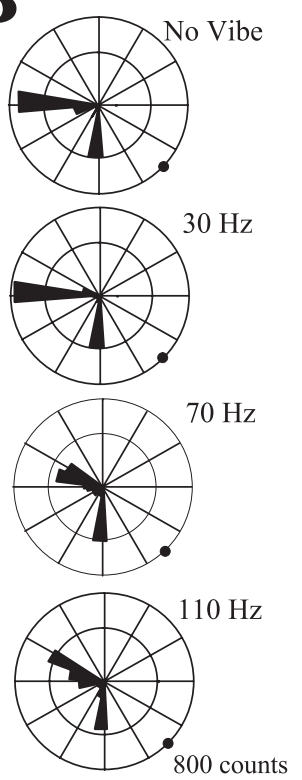

D
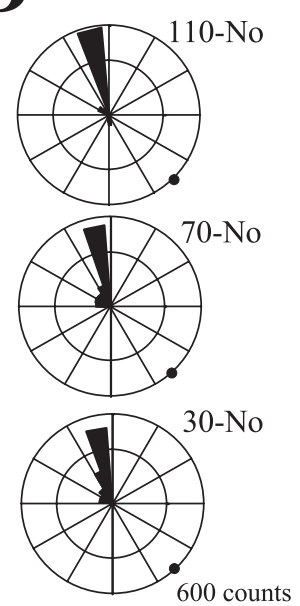

600 counts

time of this peak was $140^{\circ}$ with $110 \mathrm{~Hz}, 162^{\circ}$ at $70 \mathrm{~Hz}, 175^{\circ}$ at 30 $\mathrm{Hz}$, and $174^{\circ}$ with no vibration. The progressive shift in force direction is seen in Figure $4 \mathrm{~B}$, which shows polar histograms of the force directions during wiping trials for the four conditions. The initial cluster of forces directed to the midline of the frog (or to the left of panel) was progressively rotated rostrally toward the hip. BI vibration applied during the first $200 \mathrm{~ms}$ of wiping did not affect the magnitude or the direction of the second wave of forces. However, in some frogs, the onset of the second wave of forces was also delayed with BI vibration (see below, Independent phase regulation of a hip extensor primitive).

The altered direction of forces in the first phase shows that BI vibration did not equally enhance the activation of all muscles coactive during this period. The changes in force direction necessarily resulted from unbalanced or time-varying changes in activity of one or more of the coactive muscles, e.g., enhancing one muscle more than another. A combination of primitives are superimposed to produce the initial wave (Giszter and Kargo, 2000; Hart and Giszter, 2004). If a single primitive were to produce the initial wave of forces, or changes, the result here would indicate that BI feedback does not preserve the structure of the primitive and would present a serious challenge to the hypothesis of a set of pulsed primitives (as diagrammed in Fig. 2). A change in one primitive would be consistent with pulsed primitives but not with a single three-burst time-varying synergy for control of the trajectory. Because the isometric forces generated by individual muscles sum linearly at the ankle (Mussa-Ivaldi et al., 1994), vector subtraction of the wiping forces generated with no vibration from the time-matched forces generated during $110 \mathrm{~Hz}$ vibration should indicate one or the other alternative. If the vector subtraction produced resultant forces with varying direction over time, it would suggest an unbalanced (or differentially timevarying) effect on the ensemble of coactive muscles. However, if the subtraction produces forces that have a constant direction over time, this is consistent with amplitude scaling of one of a pair of component primitives and balanced regulation of the premotor drive and underlying muscles.

The vector subtraction procedure produced a resultant force with a constant orientation (i.e., suggesting coordinated amplitude variation of muscles consistent with Fig. 2, pattern A3). This can be seen in Figure $4 C$. The resultant forces that are produced by vector subtraction of wiping forces with no vibration from time-matched forces during 30,70, and $110 \mathrm{~Hz}$ vibration trials are shown at $40 \mathrm{~ms}$ intervals in the top panel. The resultant forces are shown only when their magnitude exceeds a threshold level (marked by the horizontal dashed line in the middle panel). The orientations of the resultant forces were invariant during the first

$\leftarrow$

two subgroups, an initial subgroup directed toward the midline of the frog and a second subgroup directed to $\sim 260^{\circ}$. The mean direction of the initial wave of forces rotated with increasing frequencies of vibration and became more dispersed (see Results), whereas the second wave of forces (after the second vertical line in $\boldsymbol{A}$ ) was not affected. $\boldsymbol{C}$, Force vectors produced by vector subtraction of the forces during no-vibration trials from the time-matched forces during 30,70 , and $110 \mathrm{~Hz}$ trials (from $\boldsymbol{A}$ ) are shown in the top panel. These are the forces added (or summed) to the wiping force pattern by BI vibration. Vertical lines indicate the period of the first wave of wiping forces. The magnitude and direction of these forces are shown in the bottom two panels (solid line represents the forces produced by $30 \mathrm{~Hz}$ with no vibration; dashed line, 70 $\mathrm{Hz}$ with no vibration; dotted line, $110 \mathrm{~Hz}$ with no vibration). Increasing the frequency of vibration enhanced the magnitude of a fixed-direction force component, which was added to the wiping force pattern. $\boldsymbol{D}$, The distribution of the resultant force directions in $\boldsymbol{C}$ (between the $\mathbf{2}$ vertical lines in C, i.e., $250 \mathrm{~ms}$ period) are shown as polar histograms. The mean direction of each distribution was similar, and the variance was low in each case (see Results). 


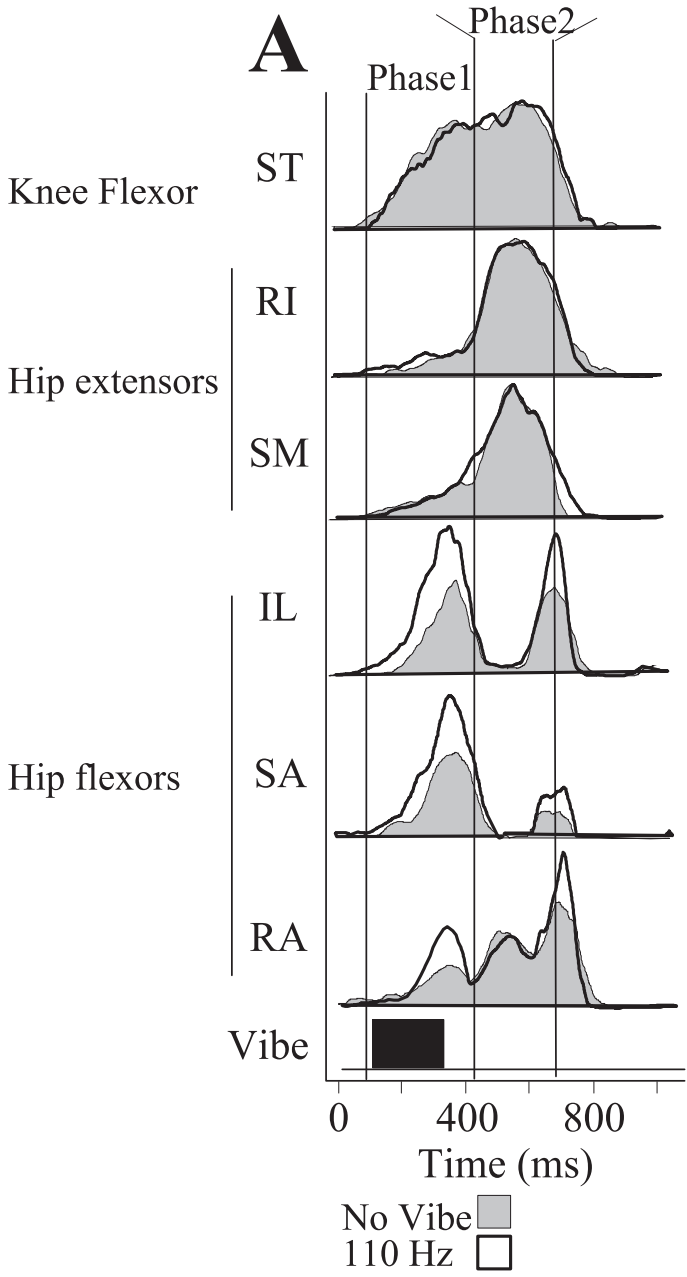

B
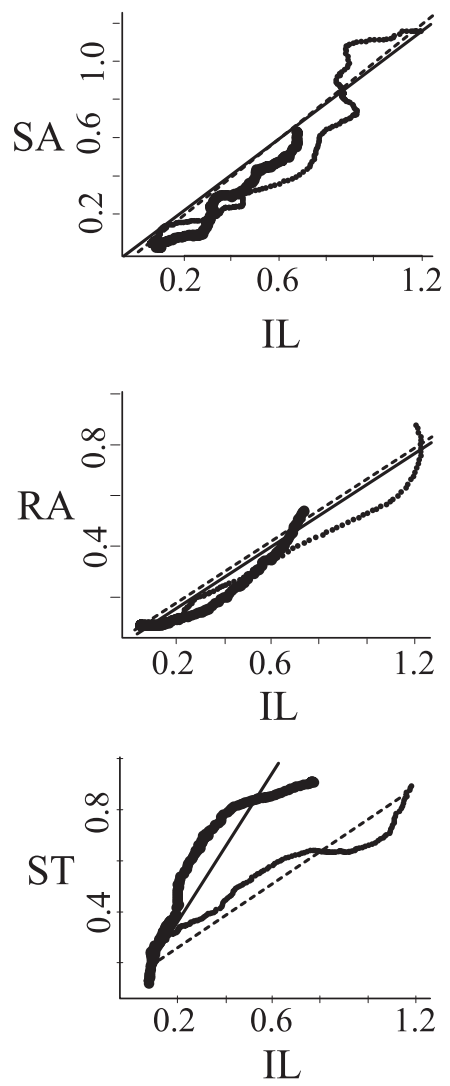

No Vibe

Figure 5. Increasing the frequency of vibration in the curarized BI enhanced the activation of a group of hip flexor muscles during the first motor phase of wiping. Data from a representative frog are shown. This result was confirmed in 10 frogs. $\boldsymbol{A}$, Rectified, filtered, and averaged EMGs are shown for six muscles (ST, RI, SM, IL, SA, and RA) during wiping without vibration (shaded envelopes) and with $110 \mathrm{~Hz}$ vibration (bold lined, unshaded envelopes). Vibration was applied for $200 \mathrm{~ms}$ at the onset of ST and is shown as a black box. Increased frequency of vibration enhanced the EMG envelopes of IL, SA, and RA but not the coactive ST muscle in the second primitive. IL, SA, and RA all flex the hip, and ST extends the hip (and also flexes the knee). $\boldsymbol{B}$, EMG -EMG plots are shown for SA versus IL, RA versus IL, and ST (knee flexor group) versus IL. Bold data lines represent EMG-EMG plots for no-vibration trials, and thinner data lines represent EMG-EMG plots for $110 \mathrm{~Hz}$ vibration. Solid and dashed straight lines represent the regression lines for the EMG-EMG plots for no vibration and $110 \mathrm{~Hz}$ vibration, respectively. For the relationships among hip flexor muscles (IL, SA, and RA), the slopes of the regression lines were not altered by vibration (i.e., the EMG increases were balanced and equal among these muscles). In contrast, the IL-ST regression line (knee flexor primitive vs hip flexor primitive) was altered by vibration (i.e., EMG increases were unbalanced or unequal).

$250 \mathrm{~ms}$ of wiping (see direction in bottom panel), and the orientation was similar for 30,70 , and $110 \mathrm{~Hz}$ vibration $\left(\sim 110^{\circ}\right)$, although magnitude altered and was scaled in a frequencydependent manner by BI feedback. This invariance can be seen to the right, in the polar histograms of the directions of the three sets of resultant forces (Fig. 4D, 30-No, 70-No, 110-No). The median bin for each of the histograms was similar $\left(108^{\circ}\right)$, and the directional variance was low $\left(<12^{\circ}\right)$. Such a low directional variance results from covarying regulation of activation of multiple muscles (Giszter et al., 1993). A low directional variance forms a criterion that we used for defining a primitive (Kargo and Giszter, 2000a) and is consistent with fixed premotor drive structures.

BI vibration effectively added a fixed-direction force pattern. Frequency of BI vibration regulated the amplitude of a component primitive to modify the initial wave of forces during wiping. Summation of this amplitude-modulated primitive with the other underlying forces resulted in the rotation of force direction. This result was thus similar in some regards to correction responses triggered cutaneously (Kargo and Giszter, 2000a).

We next examined the EMG patterns underlying this response. EMGs of target muscles were expected to covary (Fig. 2, example A3). Figure $5 A$ shows that BI vibration enhanced the activation of a specific group of muscles that were normally recruited during the first EMG phase of wiping. In this figure, rectified, filtered, and averaged EMGs are shown for six different muscles during wiping in a single frog. Shaded envelopes represent EMGs without vibration, and unshaded envelopes represent EMGs during $110 \mathrm{~Hz}$ vibration. The $110 \mathrm{~Hz}$ vibration enhanced the amplitude of three coactive muscles during the first phase of wiping (IL, SA, and RA; EMG integrals each significantly increased at $p<0.01)$. Each of these muscles generates a hip flexor torque (Loeb et al., 1999; Kargo and Rome, 2002). This same group of muscles is responsible for the trajectory correction response (Kargo and Giszter, 2000). ST, a knee flexor/hip extensor, which is also coactive during the first phase but forms part of a second primitive or independent premotor drive group (Kargo and Giszter, 2000a; Hart and Giszter, 2004), was not affected.

The summation of an unaffected ST/ knee flexor primitive with the modulation of the IL, SA, RA hip flexor primitive caused the net total force rotation discussed above. BI vibration produced balanced increases in muscle activation for each of the hip flexor-related muscles. We showed this directly by correlating the EMG waveforms of the muscle over time and at different frequencies of vibration. EMG-EMG plots are shown in Figure $5 B$ for SA-IL, RA-IL, and ST-IL. The plots start at IL EMG onset and continue up to the peak IL EMG for trials without vibration and for trials with $110 \mathrm{~Hz}$ vibration. Similar slopes across trials indicate fixed covariation of the muscles. The slope of the regression lines describing the covariation between SA and IL EMGs and between RA and IL EMGs was similar for no vibration and for $110 \mathrm{~Hz}$ vibration. The almost identical slope shows that the balance of muscle activation was not altered by BI vibration and therefore that recruitment gain and enhancement of amplitude was similar between IL, SA, and RA. Conversely, the slope of the regression line describing the covariation between IL and ST EMGs for no vibration trials differed from the slope of the regression line at $110 \mathrm{~Hz}$. Thus, this strongly suggests that IL, SA, and RA were scaled as a single control group by BI vibration and that coactive ST activity was regulated separately and independently. Because a single burst or pulse was regulated independently, we can eliminate the possibility of a three-burst time-varying synergy (Fig. 2 B1). At this point in our analysis, it remained possible 


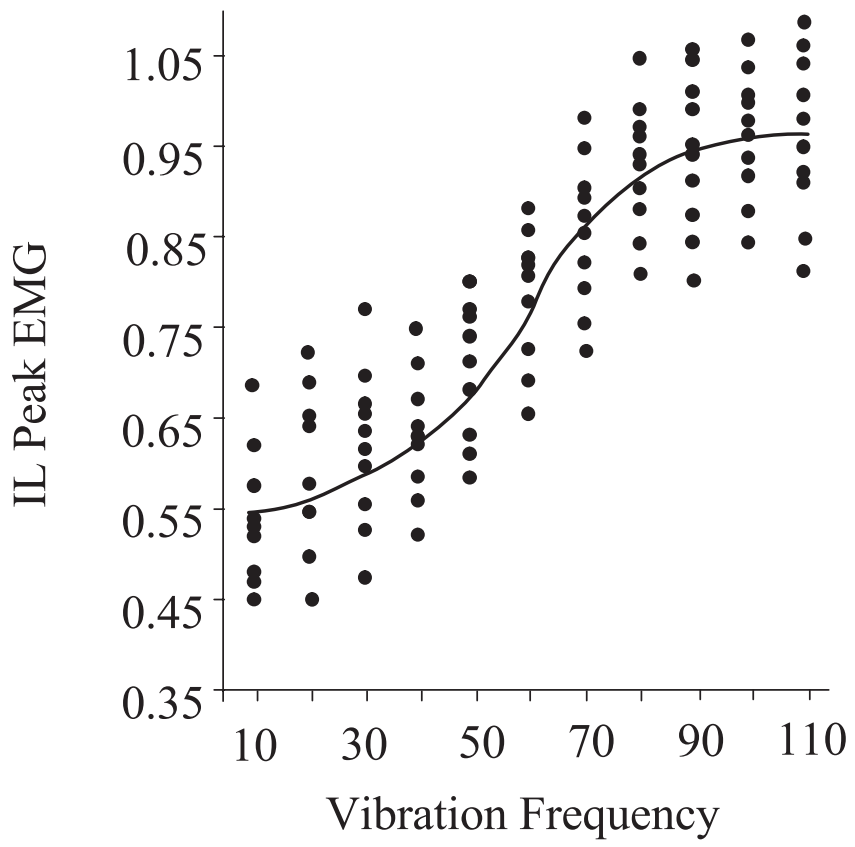

Figure 6. Enhancement of the IL hip flexor EMG burst showed a sigmoidal relationship with increasing BI vibration frequency. A scatter plot of the peak IL EMG (during the first wiping motor phase) versus vibration frequency is shown for three frogs in which vibration was applied at a range of frequencies (from 10 to $110 \mathrm{~Hz}$ by $10 \mathrm{~Hz}$ increments). Bl was curarized and vibrated for the first $200 \mathrm{~ms}$ of wiping from the onset of semitendinosus EMG in each frog. The IL EMG for individual trials in each frog was normalized to the maximum IL EMG. Each dot represents the normalized IL EMG peak for a single wiping trial at the particular applied frequency. BI vibration became effective at increasing the IL peak EMG at $\sim 50 \mathrm{~Hz}$. Effects started to saturate at $\sim 90$ $\mathrm{Hz}$.

that ST activity nonetheless formed part of a two-burst timevarying synergy with muscles RI and SM. However, these muscles will be shown to also be separable from ST by vibration in the experiments below (see Independent phase regulation of a hip extensor primitive).

\section{Vibration frequency-dependent effects on the hip flexor primitive}

In three frogs, we quantified the relationship between vibration frequency and hip flexor enhancement in more detail. In these frogs, BI was vibrated throughout the motor pattern at constant rates ranging between 10 and $110 \mathrm{~Hz}$, in $10 \mathrm{~Hz}$ increments, on different trials. Different frequency trials were interspersed randomly. Three to four trials were evoked at each frequency per frog. The IL EMG for all trials for an individual frog was then normalized to the peak IL EMG. In Figure 6, a scatter plot of the normalized IL EMG amplitude versus vibration frequency is shown for the combined data. The peak IL EMG amplitude showed a sigmoidal relationship with vibration frequency. At frequencies $30 \mathrm{~Hz}$ and below, the IL EMG was relatively constant. At frequencies between 40 and $80 \mathrm{~Hz}$, the IL EMG showed relatively larger changes. Finally, the IL EMG became saturated between 90 and $110 \mathrm{~Hz}$ vibration.

\section{Independent phase regulation of a hip extensor primitive}

Effects on the motor pattern timing occurred with more prolonged vibration. In six frogs, BI was curarized and vibrated at the onset of the wiping motor pattern (ST EMG) for a $600 \mathrm{~ms}$ duration. Thus, in these experiments, the applied vibration overlapped with the onset of the second EMG burst, i.e., of hip exten- sor activation, as well as the first. BI was again vibrated at a range of different frequencies in each frog, and we analyzed the effects of BI vibration on the second wave of forces and on the associated EMG burst.

Increasing the frequency of BI vibration delayed the onset of the hip extensor EMG burst and the transition to the second wave of force generation. In the top panel of Figure $7 A$, sample force vectors are shown every $40 \mathrm{~ms}$ for wiping trials in which BI was vibrated at 30,70, and $110 \mathrm{~Hz}$. The averaged magnitude and direction of these forces are shown in the middle panel. Averaged, rectified, and filtered EMGs from ST, RI, and IL for these data are shown in the bottom panel. It can be seen that 70 and $110 \mathrm{~Hz}$ vibration significantly delayed the time-to-half-peak of the RI EMG (by 50 and $200 \mathrm{~ms}$, respectively) relative to trials at $30 \mathrm{~Hz}$ vibration. The delay was associated with a complete phase shift of the EMG burst and not simply a shift in its onset. Figure $7 B$ shows that all muscles in this burst (primarily RI, SM, and AD) were shifted as a unit (matching Fig. 2A1). In additional support of this, we found that EMG patterns of covariation of these EMG bursts (from half-peak onset to half-peak offset) were not significantly different between each of the vibration conditions (Fig. $7 C)$. This pattern of adjustment is consistent with a single primitive and premotor drive burst. To summarize, the earlier bursts in the motor sequence were modulated differently from one another: the hip flexors amplitudes were modulated but NOT phase shifted, and the ST and knee flexor burst were modulated independently with a different gain and no phase shift. Neither showed any phase change like the hip extensor burst. The hip extensor burst that could have formed part of a two-burst timevarying synergy was regulated independently of the other bursts (corresponding to Fig. 2 B, test B2), eliminating the possible twoburst time-varying synergy. A set of complex warping functions would be needed to explain these data as time-varying synergies. Invoking Occam's razor, the simpler explanation is that the three bursts acted independently with respect to vibration as a control input, and not as time-varying synergies, but nonetheless behaved cohesively as independent premotor drive pulses.

\section{Time adjustments in the burst pulses and pattern}

BI vibration enhanced the amplitude of the hip flexor (IL) EMG burst during the first phase and delayed the extensor burst. These effects were mechanically cooperative. The combination of EMG effects (hip flexor enhancement and hip extensor delay) were associated with a rostral rotation of forces during the first phase, a small increase in the amplitude of forces composing the first phase, and a delay in the transition to caudally directed forces (Fig. 7A, middle panels). The net effect of BI vibration was thus to increase the strength of hip flexor components of the first wave of force production and increase the time window in which these components were unimpeded by hip extensor torques and thus able to exert their influences on the trajectory. We found that neither the direction nor the peak amplitude of forces composing the hip extensor primitive was affected by BI vibration (Fig. 6A, middle panels). Only phasing changes, not duration changes, occurred in the hip extensor primitive.

The relatively large delay of the hip extensor burst with prolonged $110 \mathrm{~Hz}$ vibration was not associated with an equally large increase in the duration of the hip flexor burst. In the six frogs examined here, the duration of the IL EMG burst (from half-peak onset to half-peak offset) was only moderately enhanced at 110 $\mathrm{Hz}$ compared with no-vibration trials (mean \pm SD, $305 \pm 25 \mathrm{~ms}$ duration with no vibration, $378 \pm 38 \mathrm{~ms}$ with $110 \mathrm{~Hz}$; significantly different at $p<0.05$ ). This result does suggest a role for 
A
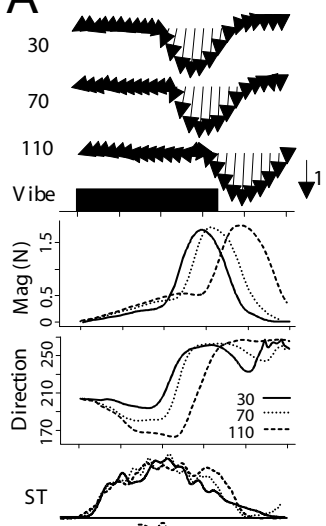

IL

$\mathrm{R}$

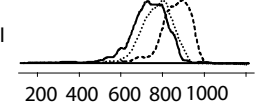

Time (ms)
B

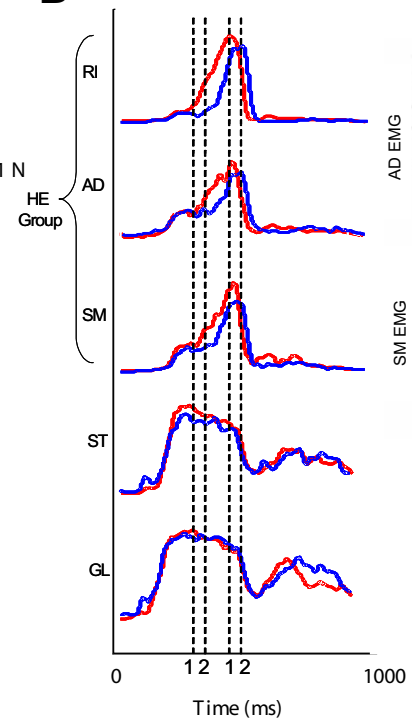

C

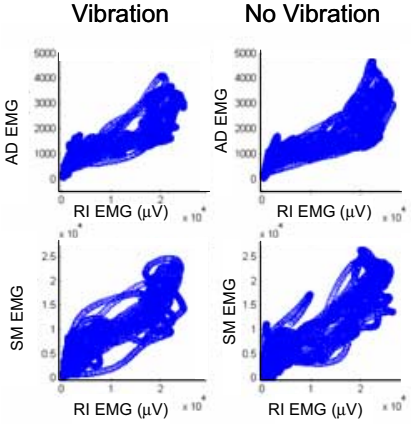

D

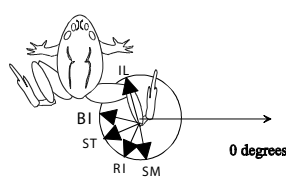

Figure 7. Increasing the frequency of vibration in the curarized BI delayed the onset of the second phase of wiping. $A$, The force vectors produced during wiping trials with 30,70 , and $110 \mathrm{~Hz}$ vibration are shown in the top panel. BI was vibrated from the onset of ST EMG for a more prolonged period (i.e., for $600 \mathrm{~ms}$; shown as a black box) than in previous experiments. This period of vibration overlapped with the second phase of wiping. The averaged magnitude and direction of the wiping forces at each vibration frequency are shown in the middle panels (solid line, $30 \mathrm{~Hz}$; dotted line, $70 \mathrm{~Hz}$; dashed line, $110 \mathrm{~Hz}$ ). The rectified, filtered, and averaged EMGs for ST, IL, and RI are shown in the bottom panel. Averages were constructed from eight trials for each frequency of vibration. Increasing the frequency of vibration delayed the onset of the second wave of (RI and SM) hip extensor forces (see diagram frog at bottom) and the associated hip extensor EMG bust (e.g., in RI). The duration of the RI EMG burst was not affected (measured from half-peak onset to half-peak offset), and therefore this response represents a phase shifting. Increasing the frequency of vibration also enhanced the amplitude of the IL EMG (and envelope area) during the initial motor phase. This IL EMG increase was associated with a rostral rotation of forces from $200^{\circ}$ at $30 \mathrm{~Hz}$ to $185^{\circ}$ at $70 \mathrm{~Hz}$ and $168^{\circ}$ at $110 \mathrm{~Hz}$. The duration of the IL EMG was increased only slightly ( $\sim 80 \mathrm{~ms})$ at the highest frequency of vibration. $\boldsymbol{B}$, A single-trial example of vibration adjusting the RI, $A D$, and SM as a unit. Dotted lines represent similar points in the waveforms; in each pair, line 1 represents no vibration, and line 2 represents vibration at $30 \mathrm{~Hz}$. C, Cross plots of the AD versus RI (above) and SM versus RI (below) for many iterations of the bursts shown in $\boldsymbol{A}$ and $\boldsymbol{B}$ for vibration (left) and for no vibration (right). Vibration causes no alterations of covariation of activity outside of the normal range of variation occurring trial to trial without vibration.

feedback in regulating the duration and time course of activation of a primitive around the mean value that was characterized by Hart and Giszter (2004). However, the delay of the hip extensor burst (of RI; calculated from ST onset to RI peak) was affected a far greater amount than the duration of the IL EMG burst. RI onset was delayed by $\sim 160 \mathrm{~ms}$ with $110 \mathrm{~Hz}$ vibration compared with $30 \mathrm{~Hz}$ vibration (mean $\pm \mathrm{SD}, 395 \pm 21 \mathrm{~ms}$ latency with no vibration, $553 \pm 29 \mathrm{~ms}$ with $110 \mathrm{~Hz}$; significantly different at $p<$ 0.01 ), but, at the same time, the RI duration was unaltered. These data thus further support independent regulations of the bursts, not a simple or uniform regulation of a time varying synergy.

\section{Vibration frequency-dependent effects on the hip extensor primitive}

In three frogs, we quantified the relationship between vibration frequency and delay of the hip extensor burst. In these frogs, BI was vibrated throughout the motor pattern at constant rates varying from $10 \mathrm{~Hz}$ up to $110 \mathrm{~Hz}$ on different trials, in $10 \mathrm{~Hz}$ increments. Low- and high-frequency trials were interspersed to prevent habituation, sensitization, and order effects. Three to four trials were evoked at each frequency per frog. The ST-to-RI latency was calculated from half-peak onset of ST to half-peak onset of RI. For each frog, the ST-to-RI latency for individual trials was normalized to the maximum latency. In Figure 8, a scatter plot of the ST-to-RI latency versus vibration frequency is shown for the combined data. We found that the ST-to-RI la- tency showed a sigmoid relationship with vibration frequency. At frequencies of vibration $50 \mathrm{~Hz}$ and below, there was little effect on the ST-to-RI latency. At frequencies between 60 and $90 \mathrm{~Hz}$, there were relatively large changes in ST-to-RI latency. Finally, at higher frequencies, the ST-to-RI latency became saturated. It is important to note that, although $\mathrm{BI}$ vibration regulated the onset of the hip extensor burst, it was not the sole determinant of hip extensor onset. Specifically, in each frog examined, prolonged periods of high-frequency vibration (1-2 s) could not delay the hip extensor burst for the duration of vibration (see below, Configuration-dependent effects of BI vibration: integration based on other measures of limb state). Thus, these data suggest that the execution timing used is a compromise between intrinsic or central pattern generator (CPG)generated burst timing and a feedbackadjusted regulation of timing in these responses.

\section{Phase-dependent effects of brief bursts of BI vibration: time-windowed effects} In the previous experimental series, we vibrated BI throughout the first phase of the motor pattern or for more prolonged periods. Thus, these experiments did not address the possible time variation or phase dependence of the effects of BI vibration. We tested the phase sensitivity of response to vibration in five frogs using brief pulses of vibration. By comparing the data with the preceding experiments that had used longer duration vibration, these experiments tested, for example, whether the effects of vibration applied during extensor muscle bursts were substantially different in form if preceded by similar vibration during the flexor phases. BI was curarized and vibrated for $100 \mathrm{~ms}$ at $100 \mathrm{~Hz}$ during the behavior. Vibration onset was triggered at different times within the wiping motor pattern. Times of vibration onset were varied systematically from $200 \mathrm{~ms}$ before ST onset to $200 \mathrm{~ms}$ after the onset of the hip extensor phase (i.e., over a $\sim 700 \mathrm{~ms}$ range of times). In subsequent analysis of responses, we measured two EMG features (Fig. 9A): (1) peak EMG of IL and (2) latency between the half-peak onset of ST and the peak of RI.

We found that our data strongly supported a phasedependent variation of the effects of muscle spindle feedback during wiping but found no qualitatively different responses. These effects are presented in Figure 9. Data for an individual frog are shown in Figure $9 B$ and for the group of five frogs in $C$. In $B$, the abscissa represents the onset time of vibration for an individual trial. The ordinate represents either the peak IL EMG (left) or the ST-to-RI latency (right). The bars above the scatter plots represent the approximate periods of unperturbed ST, IL, and RI activation in this frog (BF96). We found that periods of vibration that began around the half-peak of the ST EMG produced the largest increases in the peak IL group EMG. Thus, BI spindle volleys that occurred during the rising phase of the IL group EMG were most effective at enhancing the IL group EMG. (Note that 


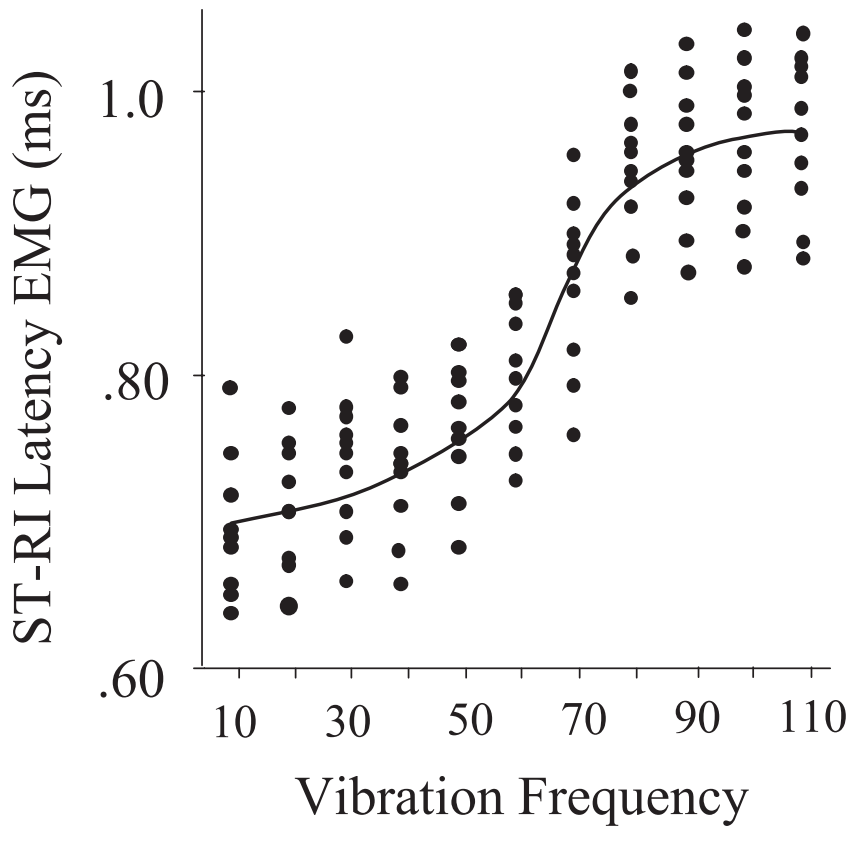

Figure 8. Delay of the hip extensor EMG burst showed a sigmoidal relationship with BI vibration frequency. A scatter plot of the ST-to-RI delay versus vibration frequency is shown for three frogs in which vibration was applied at a range of frequencies (from 10 to $110 \mathrm{~Hz}$ by $10 \mathrm{~Hz}$ increments). BI was curarized and vibrated for the first $700 \mathrm{~ms}$ of wiping from the onset of the ST EMG in each frog. The ST-to-RI delay for individual trials in each frog was normalized to the maximum delay. Each dot represents the normalized ST-to-RI delay for a single wiping trial at the particular applied frequency. BI vibration became effective at increasing the ST-to-RI delay at $\sim 60 \mathrm{~Hz}$. Effects started to saturate at $\sim 80 \mathrm{~Hz}$.

such volleys would normally be strongly enhanced and regulated by beta drive.) At other times, the effect was minimal or not significantly greater than without vibration. Conversely, we found that volleys of BI spindle action potentials that occurred 50-100 ms before the onset of the RI EMG (starting at the peak of IL and later times) were most effective at delaying the peak of the RI EMG. The consistency of these phenomena across frogs is shown in Figure 9 C.

The population data in Figure $9 C$ were obtained by combining wiping trials in which we started vibration within a similar $50 \mathrm{~ms}$ window relative to ST half-peak onset. The time window starting at the half-peak onset of the ST EMG formed the $0-50 \mathrm{~ms}$ time window for each frog. Subsequent times are also shown. These combined data show a similar pattern of effects to those seen for the individual frog in Figure $9 B$. That is, the times at which a brief duration of vibration (100 ms) caused maximal increases in the peak IL EMG and in the STto-RI delay were phasic and intermittent, spanning two peak intervals of 50-100 ms. The times of maximal effectiveness for increasing the amplitude of the hip flexor burst and for delaying the onset of the hip extensor burst were separated by $100-$ $150 \mathrm{~ms}$. Thus, there was ample possibility for setup and control of the behavior phases using spindle activity but periods of relative insensitivity to input. For example, a barrage of spindle action potentials that ended near the peak of the IL EMG would reinforce the hip flexor burst without having much effect on the onset of the hip extensor burst. These data further reinforce the notion of independently regulated pulses with differing windowed sensitivity to proprioceptive inputs.
Configuration-dependent effects of BI vibration: integration based on other measures of limb state

The previous series of experiments vibrated BI and varied its afferent feedback patterns against a background of ensemble feedback generated at a single configuration. We next tested how the results were altered if the wiping limb was not always held in a constant configuration. With the limb held at different configurations, we vibrated $\mathrm{BI}$ throughout the wipe. In this way, we tested the potential effects of configuration on the two major vibration effects described above, which could both be elicited in the same trial by the continuous vibration.

At different limb configurations and different initial muscle lengths, it is expected that the tonic and skeletofusimotor-evoked discharge of muscle spindles will vary (Murthy and Taylor, 1978; Corda et al., 1979). As a result, the ensemble background feedback might interact with the BI vibration effects in ways that the previous experiments at a fixed configuration would not detect. Thus, we positioned the wiping limb in different configurations while keeping the holding length of BI constant at each configuration. In this way, we varied the ensemble feedback in these experiments independent of BI feedback. (Note that this produced feedback that was nonphysiologic, in that the tonic firing rates of BI muscle spindles did not vary in a systematic manner with other muscle spindle populations across the configurationspace of the limb.) In these experiments, BI was either not vibrated or was vibrated continuously at $100 \mathrm{~Hz}$ through all wiping phases at each position. Positions were then compared. Seven frogs were examined.

We found that when BI was not vibrated and kept at a constant muscle length, there was a clear configuration-dependent change in the onset of the hip extensor burst. The time-to-onset of the hip extensor burst was reduced when the limb was flexed versus when the limb was extended. This resulted in an earlier transition to the second wave of extensor-directed forces from flexed limb positions. In the top row of Figure $10 A$, three successive force fields are shown at $40 \mathrm{~ms}$ intervals when BI was not vibrated and kept at a constant length. The base of each vector represents a position within the workspace of the limb (Fig. $10 \mathrm{~B}$ ). The vectors from rostral regions of the workspace showed an earlier transition to extensor-directed forces, away from midline (to orient to rostral and caudal positions, see Fig. $10 \mathrm{~B}$ ).

When BI was vibrated at $100 \mathrm{~Hz}$ at the rostral (and hip flexed) positions, the early transition to caudally directed forces seen without vibration was not apparent (see force fields in the middle row of Fig. 10A). The position-dependent effect of BI vibration was expressed as a series of force fields in the bottom row of Figure $10 \mathrm{~A}$. The fields were produced by vector subtraction of force fields generated with $100 \mathrm{~Hz}$ vibration from the fields generated without vibration. The resultant fields show that BI vibration effectively removed the early onset of extensor-directed forces usually obtained from rostral regions, i.e., flexed limb positions. The panels to the right of the force fields show that the "removal" was actually a phase delay of the second wave of forces. In the top panel, the force magnitude of two trials (solid line, no vibration; dashed line, with vibration) from a rostral position are shown. Vibration in these configurations delayed both the onset and the time of peak amplitude of the second wave of extensordirected forces. At caudal positions (bottom panel), the time of onset and peak amplitude of the second wave was not affected by $\mathrm{BI}$ vibration. In addition, we observed that the times of onset seen in caudal positions were similar to those seen from the rostral positions, when BI vibration was applied (dashed vertical line marks peak amplitude with BI vibration). 


\section{A}
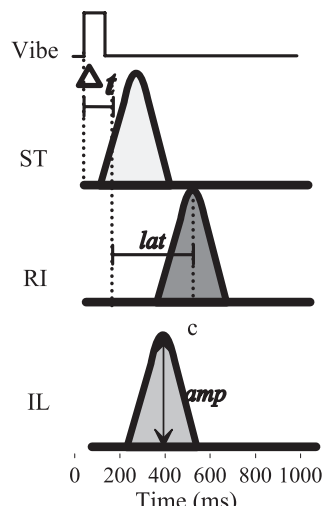

$t=$ Vibe Onset to $S T$ half peak lat $=S T$ half peak to RI peak amp=peak IL EMG

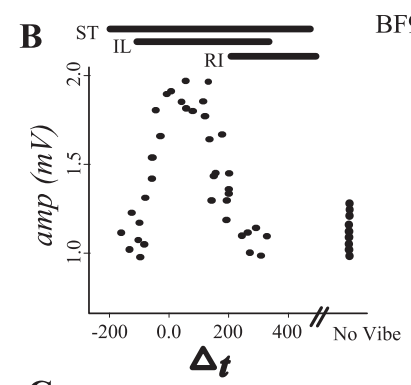

BF96
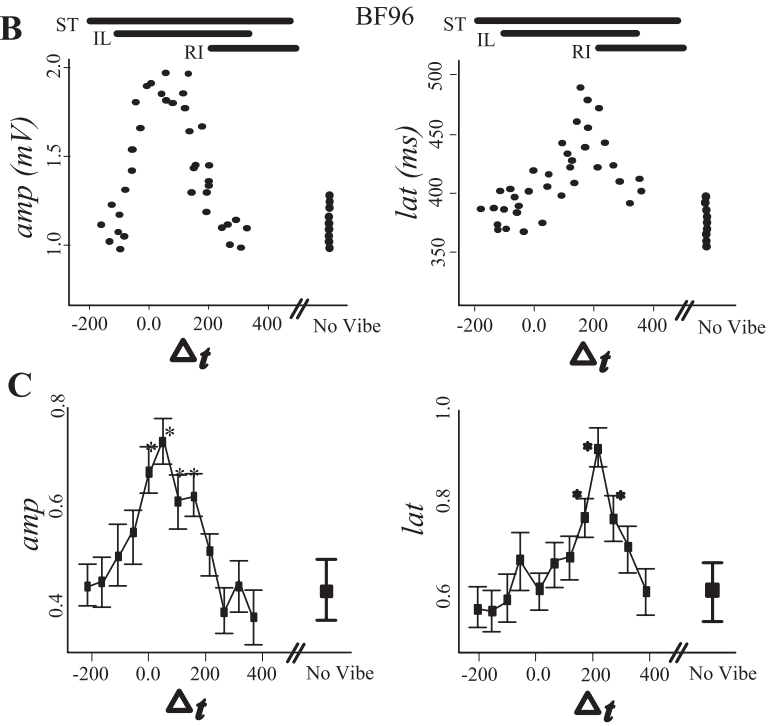

Figure 9. Brief periods of BI vibration reveal distinct effects at different times in the wiping motor pattern, i.e., episodic feedback control. In these experiments, Bl was curarized to eliminate muscle spindle activation and muscle contraction. Therefore, feedback from BI muscle afferents occurred only during the period of vibration. $A$, Schematic for the experiment is shown. The 100 ms periods of $100 \mathrm{~Hz}$ BI vibration (i.e., 10 spikes per muscle spindle; see Results) were applied at different times within the motor pattern. The motor pattern, as shown previously, consisted of an initial period of knee flexor (ST) and hip flexor (IL) activation and a second period of hip extensor activation (RI). The time of vibration onset within the motor pattern was referenced relative to the time of half-peak of ST $(\Delta t)$. The effect of the vibration for each time of application was measured as the change in the peak amplitude of IL (amp) and as the change in the latency between ST half-peak and RI peak (lat). $\boldsymbol{B}$, Data for a single frog are shown. Left, The effect of the $100 \mathrm{~ms}$ vibration on the IL peak EMG (amp) when applied at different $\Delta t$ values (each dot represents 1 trial). Above the panel are the approximate periods of ST, IL, and RI activity without BI vibration. BI vibration had the largest effects when the 100 ms vibration began 50-100 ms before the peak of the IL EMG (i.e., $\Delta t$ values from 0 to 100) and therefore overlapped with both the rising phase and peak of the IL EMG. These effects were larger than the normal variation seen when no vibration was applied to BI (right of panel). Right, The effect of brief BI vibration on the latency between ST and RI (lat). $\mathrm{BI}$ vibration had the largest effects when the $100 \mathrm{~ms}$ vibration began $50-80 \mathrm{~ms}$ before the onset of the RI EMG burst (i.e., $\Delta t$ values from 150 to 220) and therefore overlapped with a period just before and during the onset of the hip extensor burst. These effects were larger than the normal variation in lat without BI vibration. $\mathbf{C}$, Averaged data for four frogs that exhibited similar motor patterns (i.e., similar lat values of $\sim 350-400 \mathrm{~ms}$ ). For each frog, $100 \mathrm{~ms}$ Bl vibration was applied at a range of times within the motor pattern. All trials in which BI vibration was applied within a 50 ms time window starting at $\Delta t=-200$ (i.e., starting at 200-150 ms before and up to $350-400 \mathrm{~ms}$ after the half-peak of ST) were averaged together for all frogs. The averages plus SDs for the group are shown in the left and right panels for the change in both the peak IL EMG and ST-RI latency. The mean and SD of both measures when no vibration was applied is shown in the right of each panel. The time period for causing significant changes in the IL EMG peak ( $250 \mathrm{~ms}$ ) was relatively larger compared with the time period for modulating ST-RI latency (100 ms). For adjusting IL EMG amplitude, BI vibration had to be applied near the onset of the motor pattern up to the peak IL EMG. For adjusting ST-RI latency, BI vibration had to be applied near the onset of the RI EMG.

For all seven frogs, the effect of BI vibration on HE onset and HF enhancement varied across the workspace of the limb. HE onset was earliest at rostral regions when BI was not vibrated (Fig. $10 \mathrm{C}$, left panel). The $100 \mathrm{~Hz} \mathrm{BI}$ vibration produced a large delay in $\mathrm{HE}$ onset at these rostral positions. At caudal positions, the effect of vibration on HE onset was less. In the group of seven frogs, there was also a small but significant configurationdependent effect on the vibration-evoked enhancement of the IL EMG (Fig. 10D, right panel). In contrast to control of extension onset, the peak IL EMG was always enhanced at every limb configuration by BI vibration. However, the increase in the IL EMG was slightly larger when the limb was positioned in rostral regions. In rostral regions, tonic feedback from hip flexor muscles is likely to be reduced because of less muscle stretch. These results show a clear interaction between the BI spindle activity driven by vibration and the other proprioceptive information regarding limb configuration. However, despite these interactions and different controls from the BI muscle, throughout our data the general principle of superposition of three independently regulated unit premotor drive bursts as primitives for composing the motor pattern held.

\section{Discussion}

Our goals were (1) to distinguish between time-varying synergies and pulsed primitives as descriptors of execution elements in the segmental motor system and (2) to test whether spinal feedback pathways alter the structure of premotor drive or pulsed primitives that are recruited during a reflexive limb behavior (i.e., do feedback effects violate the force-field primitive framework?). To do this, we examined how a specific feedback pathway and control input (from BI muscle afferents) regulates the targeted hindlimb wiping motor pattern of spinal frogs.

\section{Feedback effects are consistent with pulsed primitives}

Our approach did not rely on statistical methods. We instead modulated wiping by vibrating the $\mathrm{BI}$ muscle and examined the changes in pattern produced and their modularity. Vibration did not disrupt premotor drive bursts or muscle balance or their associated force directions during wiping. Effects were consistent with forcefield primitives and pulsed premotor drive bursts. Feedback from BI muscle spindles independently (1) increased the amplitude of a "hip flexor" primitive and (2) regulated the phasing of a "hip extensor" primitive. Muscle spindle feedback had only a small effect on the duration of the activation of one primitive and had none on others. In contrast, there was no pairwise regulation of any of the three bursts in the targeted wiping sequence and differential sensitivity of bursts to input phase of vibration. Thus, we showed that the targets of control are single bursts, not pairs of bursts or the entire sequence. These findings match the corrections (Kargo and Giszter, 2000a) and deletions (Giszter and Kargo, 2000) in spinal frogs wiping reported previously. The data are consistent with deletions and blends in turtle (Earhart and Stein, 2000) and with deletions and corrections in cat fictive locomotion (LafreniereRoula and McCrea, 2005; Quevado et al., 2005). The new data presented here strongly support combinations of independently regulated unit bursts as composing the wiping response but do not support time-varying synergies as a spinal mechanism.

\section{The roles of feedback in frog wiping}

How proprioceptive feedback acts in frog wiping trajectories, in which primitives have frequently been characterized, is poorly understood. Brief force pulses produce little or no adjustment of motor pattern (Cheung et al., 2005). Similarly, although cutaneous inputs trigger substantial trajectory corrections, proprioceptive input alone is an ineffective trigger (Kargo and Giszter, 2000a). At the same time, it has been shown that proprioceptive deafferentation causes significantly altered trajectories and mo- 
tor patterns (Kargo and Giszter, 2000b). However, when the CNS uses reflex adjustments that are based on proprioception, it may filter inputs such that brief high-frequency transients are ignored, and responses can be highly contextual (Stein and Kearney, 1995; Kearney et al., 1997). This may reconcile these different sets of results. In this study, we use proprioceptive input generated by single muscle vibration. We tested a range of vibration patterns. These varied from $100 \mathrm{~ms}$ pulses of vibration to test phase-dependent effects, and vibration restricted to the first burst of muscle activity, to vibration that was applied continuously throughout the placing phase of the wipe. These patterns allowed us to determine how vibration effects differed during different motor bursts and if applied continuously. The durations of vibration used were all significantly longer and timed differently than the brief proprioceptive inputs likely generated by Cheung et al. (2005), in which muscle properties or "preflexes" dominated. Direct monosynaptic effects were not particularly prominent, consistent with data reported by Simpson (1969) in which group I spindle effects were all very small in fully curarized frog preparations. Conceivably, the synergistic spindle ensemble interactions we observed here and the overall excitability in the nonparalyzed frog may enhance vibration effects compared with paralyzed preparations. Responses to muscle vibration here partly match studies in mammals. Muscle vibration effects during locomotor behaviors in cats include EMG enhancement of closely related synergists and phase advance or delay of EMGs (Hiebert et al., 1996; Lam and Pearson, 2001) and the CPG (Conway et al., 1987). Vibration effects here were strongest in two different $(\sim 150 \mathrm{~ms})$ time windows, consistent with a central timing process and gating of feedback effects, when $100 \mathrm{~ms}$ pulses of vibration were tested. Additionally, BI feedback modulated the wiping motor pattern most strongly in certain limb configurations. Feedback effects depended on the context of other feedback in which the BI feedback occurred. The effects observed here match the predictions from the losses observed after lesion of proprioception: lesion caused diminished hip flexion and knee torque and collapse of hip extensor timing into a more synchronous pattern, an effect more pronounced in some configurations (Kargo and Giszter, 2000b). The spindle vibration patterns examined here can partly be related to data reported by Kargo (2000). In their data, spindles fired in episodic bursts during wiping. An early burst of BI spindle activity at motor onset varied strongly across the workspace. This phasic activity, combined with other afference, may contribute to identification of the limb initial con-
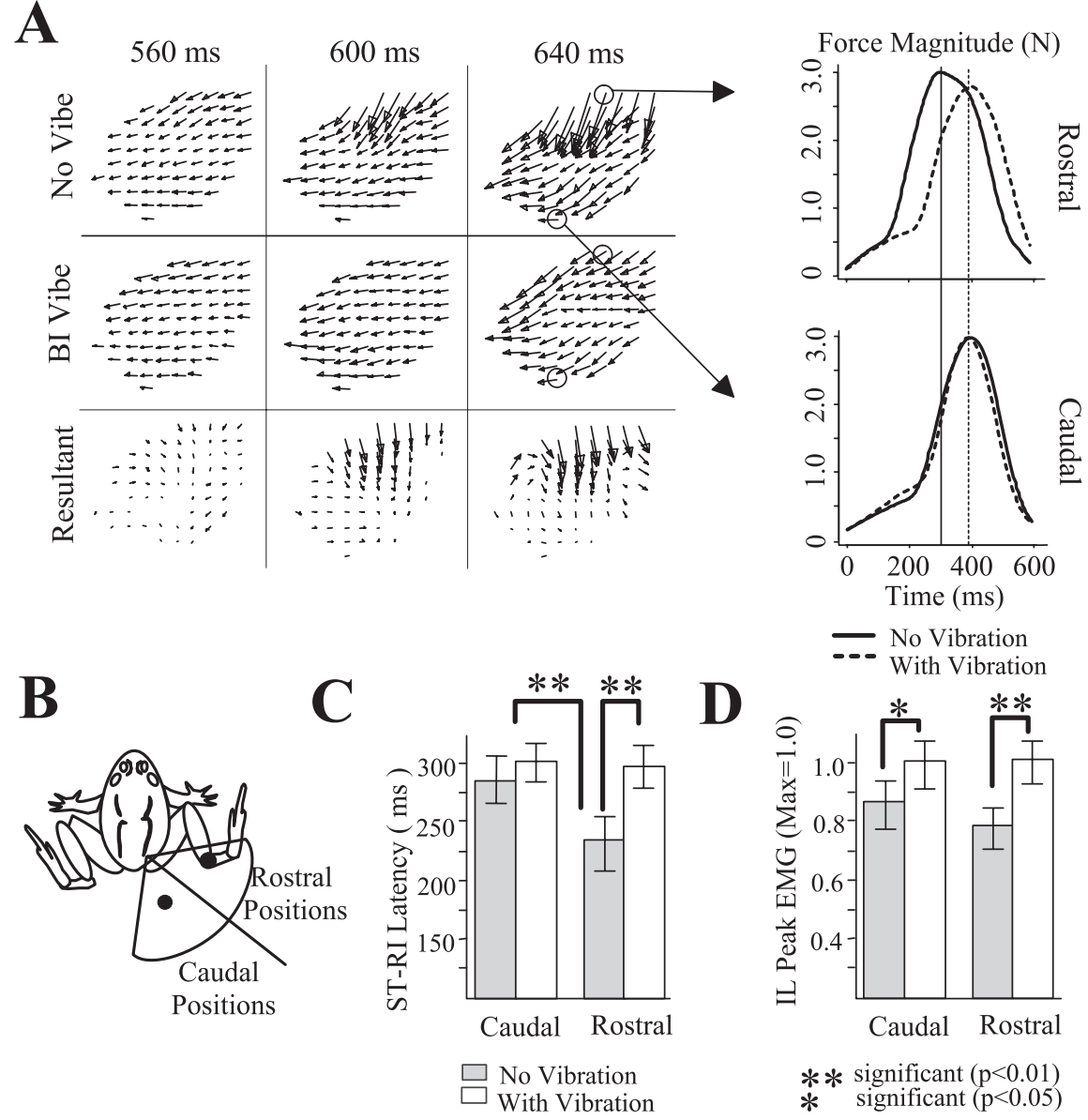

Figure 10. The effects of BI vibration vary with limb configuration. The limb was positioned at different postures, and the curarized BI muscle was kept at the same length at each posture. Either no vibration or $100 \mathrm{~Hz}$ vibration was applied at each tested posture. $A$, Force fields produced without BI vibration (top row), with Bl vibration (middle row), and the resultant fields (bottom row) produced by vector subtraction of fields with BI vibration from fields without vibration are shown. Without BI vibration, there was a position-dependent phase advance of extensor-directed forces in rostral positions (see $\boldsymbol{B}$ ). Bl vibration in rostral positions provided sensory input that would be in conflict with the postural context, because it was presumably indicated by other muscle proprioceptors. BI vibration eliminated these early extensor-directed forces when it was applied in the rostral positions. To the right of the force fields, the amplitudes of forces during wiping trials from a rostral position and a caudal position are shown (both circled on the field to the left). The solid line represents forces without BI vibration, and the dashed line represents forces with vibration. BI vibration delayed the onset of the second wave of hip extensor forces only at the rostral position and therefore acted to time-align the peak of this second wave at both rostral and caudal positions as shown in the right of $\boldsymbol{A}$ (dotted curves have aligned peaks as indicated by the dotted vertical line). $\boldsymbol{B}$, Force-field experiments were performed in seven frogs, and the workspace was divided into rostral and caudal sectors as shown. C, Bar plots show the averaged latency between ST and RI for the group of seven frogs from rostral and caudal workspace sectors and with and without vibration. Latency was calculated between the times of half-peak onset of both EMGs. BI vibration produced a significant $(p<0.01)$ increase in the ST-to-RI latency from rostral workspace positions but not in caudal positions. $D$, Bar plots show the normalized peak IL EMG for the group of seven frogs from rostral and workspace sectors and with and without vibration. BI vibration produced a significant increase in the IL peak EMG in both regions $(p<0.05)$, but the IL EMG increase was relatively larger and more significant at rostral positions. Thus, the efficacy of biceps vibration on phase delaying the hip extensor EMG burst and enhancing the hip flexor EMG burst depended on the limb configuration in which it occurred. The effect on the hip extensor burst showed a larger dependence on limb configuration than hip flexor amplitude (see Results).

ditions and parameterize the wipe pattern. The timed and gated effects of $100 \mathrm{~ms}$ bursts of BI vibration reported here resemble the gating of cutaneous inputs by Kargo and Giszter (2000a). Such gating is also common in other systems. The observation of configuration-based influences on vibration effects also mesh with frog spindle recordings: at rostral positions, the biceps vibration input will mimic the firing normally seen in more caudal extension. In the rostral limb positions, this modifies the pattern, delaying onset of hip extensor muscles. At more caudal locations, in which the other proprioceptive inputs indicate significant cau- 
dal extension, not much is added by the BI vibration. In contrast, however, the hip flexors are modulated at both locations, albeit more strongly at the caudal location. Together, the vibration effects reported are consistent with sensory adjustment of the motor pattern structure and trajectory. Our work thus suggests that an appropriate but simple regulation of independent pulses by proprioceptive feedback can organize straight isochronous targeted trajectories across the workspace of the hindlimbs.

\section{The frog as a model system}

The frog faces the same degrees of freedom problems (Bernstein, 1967) as the mammal, albeit with a simpler spindle system. The hindlimb anatomy is fairly homologous. Group Ia and group II properties of mammalian spindles are embedded in a single afferent in frogs (Husmark and Ottoson, 1970; Ottoson and Shepherd, 1970; Kirkwood, 1972). Intrafusal muscle fibers are purely beta innervated: motoneuronal branches to extrafusal muscle fibers innervate the intrafusal fibers of the spindles (Eyzaguirre, 1962). This causes obligatory "alpha-gamma" linkage: EMG fully captures the intrafusal drive. This is both a strength and limitation of the frog as a model system. Frogs allow examination of the isolated beta system, the phylogenetically more primitive condition (Houk, 1972). Simultaneously, the control rules for feedback use in phylogenetically more ancient animals might differ from those in mammals with a separated gamma system (Laporte et al., 1981).

We varied the limb configuration while holding the BI muscle length constant under isometric conditions. Under normal conditions, BI muscle length will covary with configuration-based variations in other muscle lengths as the limb moves. Golgi tendon organ activity of BI (which was avoided at the curarized muscle lengths used here) would normally also accompany some epochs of the firing of the spindle. Although biceps firing in wipes is phasic, vibration was applied in nonphysiological steps and pulses. The time-varying waveforms from neural recordings of frogs were not reproduced by us. However, manipulating feedback out of the normal range and context allow the experimenter to probe the system in novel ways that are not possible in kinematically perturbed behaviors.

\section{Hierarchy in spinal circuits}

Our data are consistent with a hierarchical model of the segmental motor system (Giszter et al., 2007; McCrea and Rybak, 2007). McCrea and Rybak propose a rhythmogenic/timing system above a pattern-shaping system. We believe that, in frogs, pattern shaping is modular and strongly structured, comprising a set of pulsed premotor drive circuits or execution primitives (Hart and Giszter, 2004). In our data, there was an overall timing influence. Feedback effects were gated, presumably by the central timing. Feedback from BI muscle was most effective in distinct windows. Such timing windows were also observed in corrections (Kargo and Giszter, 2000a). Gated reflex modulations are observed in many locomotor and motor behaviors (Clarac et al., 2000; Duysens et al., 2000).

Separable rhythmogenic circuits and pattern forming circuits have been proposed for respiration (Feldman et al., 1988), turtle scratch (Lennard, 1985), cat-paw shake (Koshland and Smith, 1989), and cat locomotion (Prochazka, 1996; Rho et al., 1999; Quevado et al., 2005; Stecina et al., 2005; McCrea and Rybak, 2007). Timed synergies have been identified in locomotion in cats (Krouchev et al., 2006) and in human studies (Cappellini et al., 2006).

\section{Hierarchy in motor planning and execution}

Several kinds of primitives have been proposed (for review, see Flash and Hochner 2005; Giszter et al., 2007). These divide into kinematic or kinetic execution elements.

Kinematic primitives have been proposed and examined in a range of ways. Kinematic strokes of specific features have been proposed as high-level or kinematic planning primitives (Viviani and Terzuolo, 1982; Hogan, 1984; Flash and Hogan, 1985; Sanger, 2000; Schaal et al., 2003; Sosnik et al., 2004; Flash and Hochner, 2005). Unit strokes or unimodal kinematic primitives, identified at the trajectory level, are used to examine adjustments and tracking (Burdet and Milner, 1998; Novak et al., 2002; Roitman et al., 2004; Fishbach et al., 2005, 2007), motor learning (Sosnik et al., 2004; Pasalar et al., 2005), and recovery of function (Doeringer and Hogan 1998; Krebs et al., 1999; Rohrer et al., 2004).

Beneath kinematic strokes must lie the appropriate joint torques and muscle activities to drive them. The spinal cord alone can construct accurate trajectories or kinematic strokes (for review, see Poppele and Bosco, 2003). The frog wiping behavior has a directed trajectory, a relatively straight path, and bell-shaped velocity profile. It is similar to human reaching in its kinematics. Frog wiping also exhibits isochrony across the workspace, similar to some cat reaching (Martin et al., 1995). Multiple premotor drive bursts or primitives contribute to the execution of these unimodal velocity profile trajectories. Groups of muscle bursts or pulses are usually needed in each kinematic stroke (Loeb 1985, Nichols 1994).

Combining muscle bursts into a single unit that matches the kinematic unit is an attractive idea (d'Avella et al., 2006; Klein Breteler et al., 2007). This "pattern chunking" idea is allied to the notion of cortical or other higher-level pattern generators (Graybiel, 1998; Klein Breteler et al., 2007). The notion of time-varying synergies represents an hypothesis about this potential chunking and provides a statistical means of examining it (d'Avella et al., 2003, 2006; d'Avella and Bizzi, 2005). Our study suggests that time-varying synergies are not the best description of the neural organization and modularity at the segmental or spinal level of motor execution. Independently regulated pulses can be shown to be the best description in this simple and experimentally tractable framework. However, as the number of pulses of muscle activity that are used in a behavior increases, to six or more for example, experiments of the kind described here can become combinatorially unwieldy. Alternate methods might be necessary to definitively disprove time-varying synergies in a more complex behavioral framework. Additionally, it is certainly conceivable that, as sequences of pulses become longer, combining controls of several pulses into one might be a natural control strategy. The strength of the data collected here, in a simpler behavior, is the feasibility of exhaustively using more direct tests and not statistical methods. However, the simpler and more tractable motor pattern examined here (which enabled such direct tests) can also be regarded as a limitation. Moreover, in thinking about these data, we emphasize that we do not imply sequence chunking or time-varying synergies are not used elsewhere in CNS. Sequences and patterns are clearly learned and organized in higher CNS centers and may be used as behavioral units. For example, conceivably, patterns organized in the brain and directly delivered to musculature via corticomotoneuronal projections might be best described in this way (Overduin et al., 2008).

Both independent pulses and sequencing are compatible if placed in a hierarchy, as described by McCrea and Rybak (2007) and Ivanenko et al. (2003) for CPGs. The frog wipe is a kinematic 
unit of behavior, comprising a directed trajectory stroke to the irritant followed by subsequent whisks, that is triggered as a unit by irritants, although independently controlled bursts support the wipe trajectory itself.

Finally, we note that the spinal primitives that support reflex trajectory generation here might "bootstrap" the development of more complex and sophisticated motor behaviors. The spinal primitives here represent phylogenetically older spinal structures for movement construction. In mammals, these structures might be used to bootstrap more sophisticated motor repertoires and skills constructed in the brain. Subsumption of spinal trajectory generating circuitry by corticospinal mechanisms could be used to circumvent the degrees of freedom problem (Giszter et al., 2000, 2001), to generate immediately useful behavior and then subsequently to elaborate a new repertoire of more complex kinematic or kinetic modules.

\section{Significance and conclusions}

We here tested whether identified heteronymous feedback would support, alter, or abolish premotor drive bursts as primitives for motor pattern construction. The data demonstrate that low-level (i.e., spinal) feedback pathway actions preserved the organization of premotor drive bursts, pulses, or force-field primitives. Independent regulation of the pulses by proprioception from the biceps muscle bolsters the idea that spinal motor patterns are constructed from independent premotor drive bursts of relatively fixed duration. The timing and amplitude of the bursts in a sequence could be independently controlled. For this reason, unitary time-varying synergies at spinal levels cannot be readily reconciled with our data.

In conclusion, our results suggest that both sensory afferent and motor efferent systems of the frog spinal cord act in combination to support and organize modular pulsed primitives for adaptive trajectory construction in the spinal cord.

\section{References}

Asada H, Slotine J-JE (1986) Robot analysis and control. New York: Wiley. Berkinblit MB, Feldman AG, Fukson OI (1986) Adaptability of innate motor patterns and motor control mechanisms. Behav Brain Sci 9:585-638.

Bernstein N (1967) The co-ordination and regulation of movements. Oxford: Pergamon.

Bizzi E, Giszter SF, Mussa-Ivaldi FA (1991) Computations underlying the execution of movement: a novel biological perspective. Science 253:287-291.

Bizzi E, Giszter SF, Loeb E, Mussa-Ivaldi FA, Saltiel P (1995) Modular organization of motor behavior in the frog's spinal cord. Trends Neurosci 18:442-446.

Bizzi E, Cheung VCK, d'Avella A, Saltiel P, Tresch M (2008) Combining modules for movement. Brain Res Rev 57:125-133.

Burdet E, Milner TE (1998) Quantization of human motions and learning of accurate movements. Biol Cybern 78:307-318.

Cappellini G, Ivanenko YP, Poppele RE, Lacquaniti F (2006) Motor patterns in human walking and running. J Neurophysiol 95:3426-3437.

Cheung VC, d'Avella A, Tresch MC, Bizzi E (2005) Central and sensory contributions to the activation and organization of muscle synergies during natural motor behaviors. J Neurosci 25:6419-6434.

Clarac F, Cattaert D, Le Ray D (2000) Central control components of a "simple" stretch reflex. Trends Neurosci 23:199-208.

Conway BA, Hultborn H, Kiehn O (1987) Proprioceptive input resets central locomotor rhythm in the spinal cord. Exp Brain Res 68:643-656.

Corda M, Pantaleo T, Calamai F (1979) Effect of motor stimulation and stretching on afferent activity of the neuromuscular spindle isolated from the frog. Arch Fisiol 71:241-263.

d'Avella A, Bizzi E (2005) Shared and specific muscle synergies in natural motor behaviors. Proc Natl Acad Sci USA 102:3076-3081.

d'Avella A, Saltiel P, Bizzi E (2003) Combinations of muscle synergies in the construction of a natural motor behavior. Nat Neurosci 6:300-308.
d'Avella A, Portone A, Fernandez L, Lacquaniti F (2006) Control of fastreaching by muscle synergy combinations. J Neurosci 26:7791-7810.

Doeringer JA, Hogan N (1998) Intermittency in preplanned elbow movements persists in the absence of visual feedback. J Neurophysiol 80:1787-1799.

Duysens J, Clarac F, Cruse H (2000) Load-regulating mechanisms in gait and posture: comparative aspects. Physiol Rev 80:83-133.

Earhart GM, Stein PS (2000) Scratch-swim hybrids in the spinal turtle: blending of rostral scratch and forward swim. J Neurophysiol 83:156-165.

Eyzaguirre C (1962) Motor regulation of the vertebrate spindle. In: Symposium on muscle receptors (Barker D, ed), pp 155-166. Hong Kong: Hong Kong UP.

Feldman JL, Smith JL, McCrimmon DR, Ellenberger HH, Speck DF (1988) Generation of respiratory patterns in mammals. In: Neural control of rhythmic movements in vertebrates (Cohen AH, Rossignol S, Grillner S, eds), pp 73-100. New York: Wiley.

Fishbach A, Roy SA, Bastianen C, Miller LE, Houk JC (2005) Kinematic properties of on-line error corrections in the monkey. Exp Brain Res 164:442-457.

Fishbach A, Roy SA, Bastianen C, Miller LE, Houk JC (2007) Deciding when and how to correct a movement: discrete submovements as a decision making process. Exp Brain Res 177:45-63.

Flash T, Hochner B (2005) Motor primitives in vertebrates and invertebrates. Curr Opin Neurobiol 15:660-666.

Flash T, Hogan N (1985) The coordination of arm movements: an experimentally confirmed mathematical model. J Neurosci 5:1688-1703.

Giszter SF, Kargo WJ (2000) Conserved temporal dynamics and vector superposition of primitives in frog wiping reflexes during spontaneous extensor deletions. Neurocomputing 32-33:775-783.

Giszter SF, Kargo WJ (2001) Modeling of dynamic controls in the frog wiping reflex: force-field level controls. Neurocomputing 38-40:1239-1247.

Giszter SF, Kargo WJ (2002) Separation and estimation of muscle spindle and tension receptor populations by vibration of the biceps muscle in the frog. Ital Archiv Biol 140:283-294.

Giszter SF, McIntyre J, Bizzi E (1989) Kinematic strategies and sensorimotor transformations in the wiping movements of frogs. J Neurophysiol $62: 750-767$.

Giszter SF, Mussa-Ivaldi FA, Bizzi E (1993) Convergent force field organized in the frog's spinal cord. J Neuroscience 13:467-491.

Giszter SF, Moxon KA, Rybak I, Chapin JK (2000) A neurobiological perspective on design of humanoid robots and their components. IEEE Intelligent Systems 15:64-69.

Giszter SF, Moxon KA, Rybak I, Chapin JK (2001) Neurobiological and neurorobotic approaches to design of a controller for a humanoid motor system. Rob Auton Syst 37:219-235.

Giszter SF, Patil V, Hart CB (2007) Primitives, premotor drives, and pattern generation: a combined computational and neuroethological perspective. Prog Brain Res 165:323-346.

Graybiel AM (1998) The basal ganglia and chunking of action repertoires. Neurobiol Learn Mem 70:119-136.

Hart CB, Giszter SF (2004) Modular premotor drives and unit bursts as primitives for frog motor behaviors. J Neurosci 24:5269-5282.

Hiebert GW, Whelan PJ, Prochazka A, Pearson KG (1996) Contribution of hind limb muscle afferents to the timing of phase transitions in the cat step cycle. J Neurophysiol 75:1126-1137.

Hogan N (1984) An organizing principle for a class of voluntary movements. J Neurosci 4:2745-2754.

Houk JC (1972) The phylogeny of muscular control configurations. In: Biocybernetics, Vol 4, pp 125-144. Jena, Germany: Fischer.

Husmark I, Ottoson D (1970) Relation between tension and sensory response of the isolated frog muscle spindle during stretch. Acta Physiol Scand 79:321-334.

Ivanenko YP, Grasso R, Zago M, Molinari M, Scivoletto G, Castellano V, Macellari V, Lacquaniti F (2003) Temporal components of the motor patterns expressed by the human spinal cord reflect foot kinematics. J Neurophysiol 90:3555-3565.

Kargo WJ (2000) Afferent regulation of force-field primitives during real limb behaviors. PhD thesis, Drexel University College of Medicine.

Kargo WJ, Giszter SF (2000a) Rapid corrections of aimed movements by combination of force-field primitives. J Neurosci 20:409-426. 
Kargo WJ, Giszter SF (2000b) Afferent roles in hindlimb wiping reflex: free limb kinematics and motor patterns. J Neurophysiol 83:1480-1501.

Kargo WJ, Rome L (2002) Functional morphology of proximal hindlimb muscles in the frog Rana pipiens. J Exp Biol 205:1987-2004.

Kearney RE, Stein RB, Parameswaran L (1997) Identification of intrinsic and reflex contributions to human ankle stiffness dynamics. IEEE Trans Biomed Eng 44:493-504.

Kirkwood PA (1972) The frequency response of frog muscle spindles under various conditions. J Physiol (Lond) 222:135-160.

Klein Breteler MD, Simura KJ, Flanders M (2007) Timing of muscle activation in a hand movement sequence. Cereb Cortex 17:803-815.

Koshland GF, Smith JL (1989) Mutable and immutable features of paw-shake responses after hindlimb deafferentation in the cat. J Neurophysiol 62:162-173.

Krebs HI, ML A, Volpe BT, Hogan N (1999) Quantization of continuous arm movements in humans with brain injury. Proc Natl Acad Sci USA 96:4645-4649.

Krouchev N, Kalaska JF, Drew T (2006) Sequential activation of muscle synergies during locomotion in the intact cat as revealed by cluster analysis and direct decomposition. J Neurophysiol 96:1991-2010.

Lafreniere-Roula M, McCrea DA (2005) Deletions of rhythmic motoneuron activity during fictive locomotion and scratch provide clues to the organization of the mammalian central pattern generator. J Neurophysiol 94:1120-1132.

Lam T, Pearson KG (2001) Proprioceptive modulation of hip flexor activity during the swing phase of locomotion in decerebrate cats. J Neurophysiol 86:1321-1332.

Laporte Y, Emonet-Denand F, Jami L (1981) The skeletofusimotor or $\beta$-innervation of mammalian muscle spindles. Trends Neurosci 5:97-99.

Lemay MA, Grill WM (2004) Modularity of motor output evoked by intraspinal microstimulation in cats. J Neurophysiol 91:502-514.

Lennard PR (1985) Afferent perturbations during "monopodal" swimming movements in the turtle: phase-dependent cutaneous modulation and proprioceptive resetting of the locomotor rhythm. J Neurosci 5:1434-1445.

Loeb E, Saltiel P, Bizzi E, Tresch M, Giszter SF (2000) Output units of motor behavior. J Cogn Neurosci 12:78-97.

Loeb GE (1985) Motoneurone task groups: coping with kinematic heterogeneity. J Exp Biol 115:137-146.

Lombard WP, Abbott FM (1907) The mechanical effects produced by the contraction of individual muscles of the thigh of the frog. Am J Physiol 20:1-60.

Martin JH, Cooper SE, Ghez C (1995) Kinematic analysis of reaching in the cat. Exp Brain Res 102:379-392.

McCrea DA, Rybak I (2007) Modeling the mammalian locomotor CPG: insights from mistakes and perturbations. Prog Brain Res 165:235-253.

Murthy KS, Taylor A (1978) Muscle spindle behavior during active muscle contraction in amphibia. Exp Neurol 60:175-188.

Mussa-Ivaldi FA (1992) From basis functions to basis fields: vector field approximation from sparse data. Biol Cybern 67:479-489.

Mussa-Ivaldi FA, Giszter SF (1992) Vector field approximation: a computational paradigm for motor control and learning. Biol Cybern 67:491-500

Mussa-Ivaldi FA. Giszter SF, Bizzi E (1994) Linear combinations of primitives in vertebrate motor control. Proc Natl Acad Sci USA 91:7534-7538.

Nichols TR (1994) A biomechanical perspective on spinal mechanisms of coordinated muscular action: an architecture principle. Acta Anat 151:1-13.

Novak KE, Miller LE, Houk JC (2002) The use of overlapping submovements in the control of rapid hand movements. Exp Brain Res 144:351-364.

Ottoson D, Shepherd GM (1970) Steps in impulse generation in the isolated muscle spindle. Acta Physiol Scand 79:423-430.

Overduin SA, d'Avella A, Roh J, Bizzi E (2008) Modulation of muscle synergy recruitment in primate grasping. J Neurosci 28:880-892.
Pasalar S, Roitman AV, Ebner TJ (2005) Effects of speeds and force fields on submovements during circular manual tracking in humans. Exp Brain Res 163:214-225.

Poppele R, Bosco G (2003) Sophisticated spinal contributions to motor control. Trends Neurosci 26:269-276.

Prochazka A (1996) Proprioceptive feedback and movement regulation. In: Handbook of physiology, Sec 12, Exercise: regulation and integration of multiple systems (Rowell LB, Shepherd JT, eds), pp 89-127. New York: American Physiological Society.

Quevedo J, Stecina K, Gosgnach S, McCrea DA (2005) Stumbling corrective reaction during fictive locomotion in the cat. J Neurophysiol 94:2045-2052.

Raasch CC, Zajac FE (1999) Locomotor strategy for pedaling: muscle groups and biomechanical functions. J Neurophysiol 82:515-525

Rho MJ, Lavoie S, Drew T (1999) Effects of red nucleus microstimulation on the locomotor pattern and timing in the intact cat: a comparison with the motor cortex. J Neurophysiol 81:2297-2315.

Robertson GA, Mortin LI, Keifer J, Stein PS (1985) Three forms of the scratch reflex in the spinal turtle: central generation of motor patterns. J Neurophysiol 53:1517-1534.

Rohrer B, Fasoli S, Krebs HI, Volpe B, Frontera WR, Stein J, Hogan N (2004) Submovements grow larger, fewer, and more blended during stroke recovery. Motor Control 8:472-483.

Roitman AV, Massaquoi SG, Takahashi K, Ebner TJ (2004) Kinematic analysis of manual tracking in monkeys: characterization of movement intermittencies during a circular tracking task. J Neurophysiol 91:901-911.

Sanger TD (2000) Human arm movements described by a low-dimensional superposition of principal components. J Neurosci 20:1066-1072.

Schaal S, Ijspeert A, Billard A (2003) Computational approaches to motor learning by imitation. Philos Trans R Soc Lond B Biol Sci 358:537-547.

Schotland JL. Rymer WZ (1993) Wipe and flexion reflexes of the frog. I. Kinematics and EMG patterns. J Neurophysiol 69:1725-1735.

Sergio LE, Ostry DJ (1993) Three-dimensional kinematic analysis of frog hindlimb movement in reflex wiping. Exp Brain Res 94:53-64.

Simpson J (1969) On why a frog is not a cat. PhD thesis, Massachusetts Institute of Technology.

Smith JL, Stein PSG (1997) Neurons, networks, and motor behavior (Stein P, Grillner S, Selverston A, Stuart D, eds), pp 47-60. Boston: Massachusetts Institute of Technology.

Sokabe M, Nunogaki K, Naruse K, Soga H, Fujitsuka N, Yoshimura A, Ito F (1993) Plateau pattern of afferent discharge rate from frog muscle spindles. J Neurophysiol 70:275-283.

Sosnik R, Hauptmann B, Karni A, Flash T (2004) When practice leads to co-articulation: the evolution of geometrically defined movement primitives. Exp Brain Res 156:422-438.

Stecina K, Quevedo J, McCrea DA (2005) Parallel reflex pathways from flexor muscle afferents evoking resetting and flexion enhancement during fictive locomotion and scratch in the cat. J Physiol (Lond) 569:275-290.

Stein PSG, McCullough ML, Currie SN (1998) Spinal motor patterns in the turtle. Ann NY Acad Sci 860:142-154.

Stein RB, Kearney RE (1995) Nonlinear behavior of muscle reflexes at the human ankle joint. J Neurophysiol 73:65-72.

Torres-Oviedo G, Macpherson JM, Ting LH (2006) Muscle synergy organization is robust across a variety of postural perturbations. J Neurophysiol 96:1530-1546.

Tresch MC, Bizzi E (1999) Responses to spinal microstimulation in the chronically spinalized rat and their relationship to spinal systems activated by low threshold cutaneous stimulation. Exp Brain Res 129:401-416.

Tresch MC, Saltiel P, Bizzi E (1999) The construction of movement by the spinal cord. Nat Neurosci 2:162-167.

Viviani P, Terzuolo C (1982) Trajectory determines movement dynamics. Neuroscience 7:431-437. 